\title{
Analytical Analysis of Effects of Buoyancy, Internal Heat Generation, Magnetic Field, and Thermal Radiation on a Boundary Layer over a Vertical Plate with a Convective Surface Boundary Condition
}

\author{
Solomon Bati Kejela (iD) and Mitiku Daba Firdi \\ Department of Mathematics, College of Natural Sciences, Jimma University, Jimma, Ethiopia \\ Correspondence should be addressed to Solomon Bati Kejela; solomonbati@yahoo.com
}

Received 29 August 2020; Revised 25 September 2020; Accepted 29 September 2020; Published 30 October 2020

Academic Editor: Ram n Quintanilla

Copyright (C) 2020 Solomon Bati Kejela and Mitiku Daba Firdi. This is an open access article distributed under the Creative Commons Attribution License, which permits unrestricted use, distribution, and reproduction in any medium, provided the original work is properly cited.

\begin{abstract}
In this paper, the effects of magnetic field, thermal radiation, buoyancy force, and internal heat generation on the laminar boundary layer flow about a vertical plate in the presence of a convective surface boundary condition have been investigated. In the analysis, it is assumed that the left surface of the plate is in contact with a hot fluid, whereas a stream of cold fluid flows steadily over the right surface, and the heat source decays exponentially outwards from the surface of the plate. The governing nonlinear partial differential equations have been transformed into a set of coupled nonlinear ordinary differential equations with the help of similarity transformation which were solved analytically by applying the optimal homotopy asymptotic method. The variations of fluid velocity and surface temperature for different values of the Grashof number, magnetic parameter, Prandtl number, internal heat generation parameter, Biot number, and radiation absorption parameter are tabulated, graphed, and interpreted in physical terms. A comparison with previously published results on similar special cases of the problem shows an excellent agreement.
\end{abstract}

\section{Introduction}

In physics and fluid mechanics, a Blasius boundary layer defines the steady two-dimensional laminar boundary layer that forms on a semi-infinite plate which is apprehended parallel to a constant unidirectional flow. By means of scaling arguments, Anderson [1] argued that about half of the terms in the Navier-Stokes equations are negligible in boundary layer flows (except in a small region near the leading edge of the plate). This leads to a condensed set of equations known as the boundary layer equations. The study on the boundary layer flow concerning a motionless plate was first done by Blasius [2].

Blasius used the similarity transformation method in the governing equation to diminish the Navier-Stoke equation for the viscous incompressible steady laminar flow over a solid boundary from the PDE to the ODE. Blasius obtained a laminar boundary layer equation
(Blasius equation) which is a third-order nonlinear ODE. The notion of the similarity solution formulated by Blasius for the boundary layer flow of a Newtonian fluid over a flat surface forms the foundation for numerous consequent studies. Later, it has been extended by various researchers [3-6] to explore the similar solutions for thermal boundary layer flows over a flat plate under altered flow configurations and boundary conditions.

In the boundary layer theory, similarity solutions were found to be valuable in the interpretation of certain fluid motions at big Reynolds numbers. Similarity solutions frequently exist for the flow over semi-infinite plates and stagnation point flow for two-dimensional, irregular, and three-dimensional bodies [7]. In exceptional cases, when there is no similarity solution, one has to solve a system of nonlinear partial differential equations. For similarity boundary-layer flows, velocity profiles are alike, but this kind of similarity is missing for nonsimilarity flows [8-11]. 
Boundary-layer flows over a moving or stretching plate are of great importance in view of their relevance to an extensive variety of technical applications, especially in the manufacture of fibers in glass and polymer industries [7]. The first and leading work regarding boundary layer behaviour in moving surfaces in a quiescent fluid was executed by Sakiadis [12]. Consequently, many researchers [13-17] worked on the problem of moving or stretching plates under different situations.

The heat transfer analysis of boundary-layer flows with radiation is also vital in electrical power generation, astrophysical flows, solar power technology, space vehicle reentry, and other industrial areas [7]. Raptis et al. [18] investigated the effect of thermal radiation on the magnetohydrodynamic flow of a viscous fluid past a semi-infinite stationary plate. Hayat et al. [19] prolonged the analysis of Raptis et al. for a second-grade fluid.

Convective heat transfer studies are very substantial in processes involving high temperatures, such as gas turbines, thermal energy storage, and nuclear plants. Ishak [20] examined the similarity solutions for flow and heat transfer over a permeable surface with a convective boundary condition. Aziz [21,22] investigated a similarity solution for the laminar thermal boundary layer over a flat plate with a convective surface boundary condition and also studied hydrodynamic and thermal slip flow boundary layers over a flat plate with a constant heat flux boundary condition. Garg et al. [23] studied a similarity solution for the laminar thermal boundary layer over a flat plate with internal heat generation and a convective surface boundary condition.

Moreover, Makinde and Oladapo Olanrewaju [24] investigated the buoyancy effects on a thermal boundary layer over a vertical plate with a convective surface boundary condition. They also investigated the effect of buoyancy force, thermal radiation, and internal heat generation by the numerical method [7]. However, to the best of our knowledge, no investigation has been made yet to analyze the effects of buoyancy, internal heat generation, thermal radiation, and magnetic parameter on a boundary layer flow over a vertical plate with a convective surface boundary condition by the analytical or semianalytical method.

Hence, the objective of this study was to investigate the combined effects of buoyancy force, internal heat generation, thermal radiation, and magnetic field on a boundary layer over a vertical plate with a convective surface boundary condition via optimal homotopy asymptotic method (OHAM).

\section{Basic Concepts of the Optimal Homotopy Asymptotic Method}

The OHAM was introduced and developed by Marinca et al. [25-27]. OHAM is a modification of the homotopy asymptotic method which is based on reducing the residual error. In this method, the control and adjustment of the convergence region are provided in a suitable way. To illustrate the basic concepts of the optimal homotopy asymptotic method, the following nonlinear differential equation is considered:

$$
A[w(x)]+b(x)=0, \quad x \in D
$$

with boundary condition

$$
B\left(w, \frac{\mathrm{d} w}{\mathrm{~d} x}\right)=0
$$

where $A$ is a differential operator, $B$ is a boundary operator, $w(x)$ is an unknown function, $D$ is the problem domain, and $b(x)$ is a known analytic function. The operator $A$ can be decomposed as $A=L+N$, where $L$ is a linear operator and $N$ is a nonlinear operator.

According to the OHAM, one can construct an optimal homotopy $\psi(x ; p): D x[0,1] \longrightarrow \mathbb{R}$ which satisfies

$$
(1-p)[L(\psi(x ; p))+b(x)]-H(p)[A(\psi(x ; p))+b(x)]=0,
$$

where $p \in[0,1]$ is an embedding parameter, $H(p)$ is a nonzero auxiliary function for $p \neq 0$, and $H(0)=0$. When $p=0 \quad$ and $\quad p=1$, we get $\quad \psi(x, 0)=w_{0}(x)$ and $\psi(x, 1)=w(x)$, respectively. Thus, as $p$ increases from 0 to 1 , the solution $\psi(x ; p)$ varies from $w_{0}(x)$ to $w(x)$, where $w_{0}(x)=\psi(x, 0)$ is obtained from the homotopy (3). For $p=0$, we have

$$
\begin{array}{r}
L\left(w_{0}(x)\right)+b(x)=0 \\
B\left(w_{0}, \frac{\mathrm{d} w_{0}}{\mathrm{~d} x}\right)=0
\end{array}
$$

The auxiliary function $H(p)$ is chosen in the form

$$
\begin{aligned}
H(p) & =\sum_{k=1}^{m} p^{k} c_{k} \\
& =c_{1} p+c_{2} p^{2}+c_{3} p^{3}+\ldots
\end{aligned}
$$

The convergence of the solution greatly depends on this function. The function $H(p)$ also adjusts the convergence domain and controls the convergence region. To obtain an approximate solution, $\psi(x ; p)=\psi\left(x ; p, c_{1}, c_{2}, c_{3}, \ldots\right)$ is expanded in a series about $p$ as

$$
\psi\left(x ; p, c_{1}, c_{2}, c_{3}, \ldots\right)=w_{0}(x)+\sum_{i=1}^{\infty} w_{i}\left(x, c_{1}, c_{2}, c_{3}, \ldots\right) p^{i}
$$

Substituting equation (6) into equation (3) and equating the coefficient of like powers of $p$, the zero-order problem is obtained as given in equation (4). The first- and secondorder problems are, respectively, given by equations (7) and (8): 


$$
\begin{aligned}
L\left(w_{1}(x)\right) & =c_{1} N_{0}\left(w_{0}(x)\right), \\
B\left(w_{1}, \frac{\mathrm{d} w_{1}}{\mathrm{~d} x}\right) & =0, \\
L\left(w_{2}(x)\right)-L\left(w_{1}(x)\right)= & c_{2} N_{0}\left(w_{0}(x)\right) \\
& +c_{1}\left[L\left(w_{1}(x)\right)\right. \\
& \left.+N_{1}\left(w_{0}(x) ; w_{1}(x)\right)\right], \\
B\left(w_{2}, \frac{\mathrm{d} w_{2}}{\mathrm{~d} x}\right)= & 0 .
\end{aligned}
$$

Hence, the general governing equations for $w_{j}(x)$ are given by

$$
\begin{aligned}
L\left(w_{j}(x)\right)-L\left(w_{j-1}(x)\right)= & c_{j} N_{0}\left(w_{0}(x)\right) \\
& +\sum_{i=1}^{j-1} c_{i}\left[L\left(w_{j-i}(x)\right)\right. \\
& \left.+N_{j-i}\left(w_{0}(x), w_{1}(x), \ldots, w_{j-1}(x)\right)\right] \\
B\left(w_{j}, \frac{\mathrm{d} w_{j}}{\mathrm{~d} x}\right)= & 0, \quad j=2,3, \ldots,
\end{aligned}
$$

where $N_{m}\left(w_{0}(x) ; w_{1}(x) ; w_{2}(x) ; \ldots ; u_{m}(x)\right)$ is the coefficient of $p^{m}$ in the expansion of $N\left(\psi\left(x ; p, c_{1}, c_{2}, c_{3}, \ldots\right)\right)$ with respect to the embedding parameter $p$ as follows:

$$
\begin{aligned}
N\left(\psi\left(x ; p, c_{1}, c_{2}, c_{3}, \ldots\right)\right)= & N_{0}\left(w_{0}(x)\right) \\
& +\sum_{j=1}^{\infty} N_{j}\left(w_{1}, w_{2}, w_{3}, \ldots, w_{j}\right) p^{j},
\end{aligned}
$$

where $\psi\left(x ; p, c_{1}, c_{2}, c_{3}, \ldots\right)$ is given in equation (6). It should be underlined that $w_{j}$ for $j \geq 0$ are governed by the linear equations with linear boundary conditions that come from the original problem, which can be smoothly solved. Moreover, the convergence of the series given in equation (6) depends upon the convergence-control parameters, $c_{1}, c_{2}, c_{3}, c_{4}, \ldots$

If the series converges at $p=1$, one has

$$
w\left(x, c_{1}, c_{2}, c_{3}, \ldots\right)=w_{0}(x)+\sum_{j=1}^{\infty} w_{j}\left(x, c_{1}, c_{2}, c_{3}, \ldots\right) .
$$

The general solution of equation (1) can be determined approximately in the form

$$
w^{m}\left(x, c_{1}, c_{2}, c_{3}, \ldots\right)=w_{0}(x)+\sum_{j=1}^{m} w_{j}\left(x, c_{1}, c_{2}, c_{3}, \ldots, c_{m}\right) .
$$

Substituting equation (12) in equation (1), we get the following expression for the residual error:

$$
\begin{array}{r}
R\left(x, c_{i}\right)=L\left(w^{m}\left(x, c_{i}\right)\right)+b(x)+N\left(w^{m}\left(x, c_{i}\right)\right), \\
i=1,2,3, \ldots, m .
\end{array}
$$

If $R\left(x, c_{i}\right)=0$, then $w^{m}\left(x, c_{i}\right)$ is the exact solution of the given equation, but this does not happen, especially in nonlinear problems.

The optimal values of the convergence-control parameters $\left(c_{i}\right)$ can be identified via various methods, such as the least square method, the Galerkin method, the collocation method, the Ritz method, and the Kantorovich method. In order to find the optimal values of $c_{1}, c_{2}, c_{3}, c_{4}, \ldots$ by the least square method, we first construct functional

$$
J\left(c_{i}\right)=\int_{a}^{b} R^{2}\left(x, c_{i}\right) \mathrm{d} x,
$$

and then minimize it, where $a$ and $b$ are in the domain of the given problem and $R$ is given by equation (13). The unknown convergence-control parameters $c_{i}, i=1,2,3, \ldots, m$, can be identified from the following condition:

$$
\frac{\partial J}{\partial c_{i}}=0, \quad i=1,2,3, \ldots, m .
$$

With these parameters known, the $m^{\text {th }}$-order approximate solution given by equation (12) is well determined. The least square method is a powerful technique and has been used in many other methods such as the optimal auxiliary function method (OAFM), optimal homotopy perturbation method (OHPM), and optimal homotopy asymptotic method (OHAM) with Daftardar-Jeffery polynomials (OHAM-DJ) for calculating the optimum values of arbitrary constants [28-30].

\section{Mathematical Formulation}

A two-dimensional steady incompressible fluid flow coupled with heat transfer by convection over a vertical plate in a stream of cold fluid at temperature $T_{\infty}$ which moved over the right surface of the plate with a uniform velocity $U_{\infty}$, whereas the left surface of the plate was heated by convection from a hot fluid at temperature $T_{f}$ which provided a heat transfer coefficient $h_{f}$, is considered. The density difference as a result of buoyancy force influences and the effect of the magnetic field were taken into account in the momentum equation; the internal heat generation and the thermal radiation effects were taken into description in the energy equation (the Boussinesq approximation). The continuity, momentum, and energy equations describing the flow are summarized by the following boundary value problem $[21,24]$ :

$$
\begin{gathered}
\frac{\partial u}{\partial x}+\frac{\partial v}{\partial y}=0 \\
u \frac{\partial u}{\partial x}+v \frac{\partial u}{\partial y}=v \frac{\partial^{2} u}{\partial y^{2}}+g \beta\left(T-T_{\infty}\right)-\frac{\delta}{\rho} B_{0}^{2} u
\end{gathered}
$$




$$
u \frac{\partial T}{\partial x}+v \frac{\partial T}{\partial y}=\frac{k}{\rho c_{p}} \frac{\partial^{2} T}{\partial y^{2}}-\frac{1}{\rho c_{p}} \frac{\partial q_{r}}{\partial y}+\frac{Q}{\rho c_{p}}\left(T-T_{\infty}\right),
$$

with the boundary conditions defined as follows:

$$
\begin{aligned}
u(x, 0) & =v(x, 0)=0, \\
-k \frac{\partial T}{\partial y}(x, 0) & =h_{f}\left[T_{w}-T(x, 0)\right], \\
y & =\infty \\
u & \longrightarrow u_{\infty}, \\
T(x, \infty) & =T_{\infty}, \\
y & \longrightarrow \infty
\end{aligned}
$$

where $u$ and $v$ are the velocity components along the flow direction ( $x$-direction) and normal to the flow direction $(y$ direction), $v$ is the kinematic viscosity, $\beta$ is the thermal volumetric expansion coefficient, $g$ is the gravitational acceleration, $k$ is the thermal conductivity, $c_{p}$ is the specific heat of the fluid at constant pressure, $T$ is the temperature of the fluid inside the thermal boundary layer, $\rho$ is the density, $q_{r}$ is the radiative heat flux in the $y$-direction, $u_{\infty}$ is a constant free-stream velocity, $\delta$ is the electrical conductivity of the base fluid, $B_{0}$ is the imposed magnetic field, $T_{\infty}$ is the constant temperature of the ambient fluid, $Q$ is the heat released per unit per mass, and $h_{f}$ is the heat transfer coefficient.

It is assumed that the viscous dissipation is neglected, the physical properties of the fluid are constant, and the Boussinesq and boundary layer approximation may be adopted for steady laminar flow. The radiative heat flux $q_{r}$ is described by Rosseland approximation as

$$
q_{r}=-\frac{4 \sigma^{*}}{3 k^{\prime}} \frac{\partial T^{4}}{\partial y}
$$

where $k^{\prime}$ and $\sigma^{*}$ are the mean absorption coefficient and the Stefan-Boltzmann constant, respectively. Suppose the temperature variations within the flow are adequately small so that $T^{4}$ can be expressed as a linear function after using Taylor series to expand $T^{4}$ about the free stream temperature $T_{\infty}$ and ignoring higher-order terms. This results in the following approximation:

$$
T^{4} \cong 4 T_{\infty}^{3} T-3 T_{\infty}^{4}
$$

On using equation (22) in equation (21), we have

$$
q_{r}=\frac{-16 \sigma^{*} T_{\infty}^{3}}{3 k^{\prime}} \frac{\partial T}{\partial y}
$$

Plugging equation (23) into equation (18), we get the following equation:

$$
u \frac{\partial T}{\partial x}+v \frac{\partial T}{\partial y}=\left(\alpha+\frac{16 \sigma^{*} T_{\infty}^{3}}{3 k^{\prime}} \cdot \frac{\alpha}{k}\right) \frac{\partial^{2} T}{\partial y^{2}}+\frac{\alpha}{k} Q\left(T-T_{\infty}\right)
$$

where $\alpha=k / \rho c_{p}$ is the thermal diffusivity; from this equation, it is clearly seen that the effect of radiation is to enhance the thermal diffusivity. If we take $N_{R}=k k^{\prime} / 4 \sigma^{*} T_{\infty}^{3}$ as the radiation parameter in equation (24), the equation becomes

$$
u \frac{\partial T}{\partial x}+v \frac{\partial T}{\partial y}=\frac{\alpha}{k_{o}} \cdot \frac{\partial^{2} T}{\partial y^{2}}+\frac{\alpha}{k} Q_{o}\left(T-T_{\infty}\right),
$$

where $k_{o}=3 N_{R} / 3 N_{R}+4$. It is clear that the classical solution for the energy equation without the thermal radiation influence can be obtained from equation (25), which reduces to the following equation:

$$
u \frac{\partial T}{\partial x}+v \frac{\partial T}{\partial y}=\alpha \frac{\partial^{2} T}{\partial y^{2}}+\frac{\alpha}{k} Q_{o}\left(T-T_{\infty}\right) .
$$

A similarity variable $\eta$ and dimensionless stream function $f(\eta)$ are introduced as follows:

$$
\begin{aligned}
\eta & =y \sqrt{\frac{u_{\infty}}{v x}}, \\
\frac{u}{u_{\infty}} & =f^{\prime}, \\
v & =\frac{1}{2} \sqrt{\frac{u_{\infty} v}{x}}\left(\eta f^{\prime}-f\right) .
\end{aligned}
$$

Differentiating $u$ and $v$ with respect to $x$ and $y$, respectively, we get

$$
\begin{aligned}
& \frac{\partial u}{\partial x}=-\frac{u_{\infty} f^{\prime \prime} \eta}{2 x} \\
& \frac{\partial v}{\partial y}=\frac{u_{\infty} \eta f^{\prime \prime}}{2 x}
\end{aligned}
$$

where $f^{\prime}=\mathrm{d} f / \mathrm{d} \eta$ and $u_{\infty}$ is a constant free-stream velocity. Substituting equation (28) into equation (15), the equation of continuity is satisfied identically. From equation (27), we can also obtain:

$$
\begin{aligned}
& \frac{\partial u}{\partial y}=u_{\infty} f^{\prime \prime} \sqrt{\frac{u_{\infty}}{v x}} \\
& \frac{\partial^{2} u}{\partial y^{2}}=\frac{u_{\infty}^{2}}{v x} f^{\prime \prime \prime}
\end{aligned}
$$

The nondimensional form of the temperature $\theta(\eta)$ is defined as follows:

$$
\theta(\eta)=\frac{T-T_{\infty}}{T_{w}-T_{\infty}}
$$

where $T_{w}$ is a constant temperature of the wall.

Substituting equations (27)-(30) into equation (17), we obtain

$$
f^{\prime \prime \prime}+\frac{1}{2} \mathrm{ff}^{\prime \prime}+\mathrm{Gr}_{x} \theta-\mathrm{Mf}^{\prime}=0
$$

where 


$$
\begin{aligned}
G r_{x} & =\frac{g \beta\left(T w-T_{\infty}\right) x}{u^{2} \infty}, \\
M^{2} & =\frac{\delta B_{0}^{2} x}{\rho u_{\infty}} .
\end{aligned}
$$

$G r_{x}$ is the local Grashof number, and $M$ is the magnetic parameter.

From equation (30), we have

$$
\begin{gathered}
\frac{\partial T}{\partial x}=-\frac{\theta^{\prime} \eta}{2 x}\left(T_{w}-T_{\infty}\right), \\
\frac{\partial^{2} T}{\partial y^{2}}=\theta^{\prime \prime} \frac{u_{\infty}}{v x}\left(T_{w}-T_{\infty}\right) .
\end{gathered}
$$

Substituting equations (27)-(30) and (33) into equation (25), we get

$$
\theta^{\prime \prime}+k_{o} \operatorname{Pr}\left(\frac{1}{2} f \theta^{\prime}+\lambda_{x} \theta(\eta)\right)=0
$$

with the boundary conditions

$$
\begin{aligned}
\theta^{\prime}(0) & =-\mathrm{Bi}_{x}[1-\theta(0)], \\
\theta(\infty) & =0,
\end{aligned}
$$

where $\operatorname{Pr}=v / \alpha$ is the Prandtl number, $\lambda_{x}=Q_{o} x / u_{\infty} \rho c_{p}$ is the internal heat, and

$$
\mathrm{Bi}_{x}=\frac{h_{f}}{k} \sqrt{\frac{v x}{u_{\infty}}} .
$$

Equations (19) and (27) give the transformed boundary conditions for velocity fields as follows:

$$
f(\eta)=f^{\prime}(\eta)=0, \quad \eta=0 .
$$

For the momentum and energy equations to have a similarity solution, the parameters $\mathrm{Gr}_{x}, \lambda_{x}, M^{2}$, and $\mathrm{Bi}_{x}$ must be constants and not functions of $x$ as in equations (32) and (36). This condition can be met if the heat transfer coefficient $h_{f}$ is proportional to $1 / \sqrt{x}$ and the thermal expansion coefficient is proportional to $1 / x$. We therefore assume

$$
\begin{aligned}
h_{f} & =c x^{-1 / 2}, \\
\beta & =m x^{-1}, \\
B_{0}^{2} & =d^{2} x^{-1}, \\
Q & =t x^{-1},
\end{aligned}
$$

where $c, t, d$, and $m$ are constants.

Putting equation (38) into equations (32) and (36), we get

$$
\begin{aligned}
\mathrm{Gr} & =\frac{m g v\left(T_{w}-T_{\infty}\right)}{u_{\infty}^{2}}, \\
\lambda & =\frac{t}{u_{\infty} \rho c_{p}}, \\
M & =\sqrt{\frac{\delta}{\rho u_{\infty}}} d, \\
B i & =\frac{c}{k} \sqrt{\frac{v}{u_{\infty}}}
\end{aligned}
$$

with $G r, \lambda$, and $M$ being defined by equation (39); the solutions of equation (40) with boundary condition equation (41) produce similarity solutions. Equations (20) and (27) suggested that $f^{\prime}(\eta)=1$ as $\eta \longrightarrow \infty$.

Thus, the transformed equations representing the flow problem with their respective boundary conditions are expressed in equations (40) and (41):

$$
\begin{aligned}
& \left\{\begin{array}{l}
f^{\prime \prime \prime}+\frac{1}{2} f f^{\prime \prime}+G r \theta-M f^{\prime}=0, \\
\theta^{\prime \prime}+k_{o} \operatorname{pr}\left(\frac{1}{2} f \theta^{\prime}+\lambda \theta\right)=0,
\end{array}\right. \\
& \left\{\begin{array}{l}
f(\eta)=f^{\prime}(\eta)=0, \\
\theta^{\prime}(\eta)=-B i[1-\theta(0)], \quad \eta=0, \\
f^{\prime}(\eta)=1, \theta(\eta)=0, \quad \eta \longrightarrow \infty .
\end{array}\right.
\end{aligned}
$$

\section{Solution of the Problem}

Applying the OHAM on equation (40), we can construct the following homotopy:

$$
\left.\begin{array}{l}
(1-p)\left(f^{\prime \prime \prime}\right)-H_{1}(p)\left[f^{\prime \prime \prime}+\frac{1}{2} f f^{\prime \prime}+\mathrm{Gr} \theta-M f^{\prime}\right]=0 \\
(1-p)\left(\theta^{\prime \prime}\right)-H_{2}(p)\left[\theta^{\prime \prime}+k_{o} \operatorname{Pr}\left(\frac{1}{2} f \theta^{\prime}+\lambda \theta(\eta)\right)\right]=0
\end{array}\right\},
$$

where $p \in[0,1]$ and the primes denote differentiation of the function $f$ with respect to $\eta ; f, \theta, H_{1}$, and $H_{2}$ are considered as follows:

$$
\begin{aligned}
f & =f_{0}+p f_{1}+p^{2} f_{2}+p^{3} f_{3}, \\
\theta & =\theta_{0}+p \theta_{1}+p^{2} \theta_{2}+p^{3} \theta_{3}, \\
H_{1}(p) & =p c_{1}+p^{2} c_{2}+p^{3} c_{3}, \\
H_{2}(p) & =p c_{4}+p^{2} c_{5}+p^{3} c_{6} .
\end{aligned}
$$


Using equation (43) in equation (42), simplifying, and equating the coefficients of the same powers of $p$, we get the following set of zeroth-, first-, second-, and third-order problems.

The zeroth-order problems are

$$
\begin{gathered}
f_{0}^{\prime \prime}(\eta)=0, \\
\theta_{0}^{\prime \prime}(\eta)=0,
\end{gathered}
$$

subjected to boundary conditions

$$
\begin{aligned}
f_{0}(0) & =f_{0}^{\prime}(0)=0, f_{0}^{\prime}(\infty)=1, \\
\theta_{0}^{\prime}(0) & =-\operatorname{Bi}(1-\theta(0)), \theta_{0}(\infty)=0 .
\end{aligned}
$$

The first-order problems are

$$
\left.\begin{array}{l}
f_{1}^{\prime \prime}\left(\eta, c_{1}\right)=c_{1}\left(\frac{1}{2} f_{0} f_{0}^{\prime \prime}+G r \theta_{0}-M f_{0}^{\prime}\right) \\
\theta_{1}^{\prime \prime}\left(\eta, c_{1}\right)=c_{4} \operatorname{Pr}\left(\frac{1}{2} k_{0} f_{0} \theta_{0}^{\prime}+\lambda k_{0} \theta_{0}\right)
\end{array}\right\},
$$

subjected to boundary conditions

$$
f_{1}(0)=f_{1}^{\prime}(0)=f_{1}^{\prime}(\infty)=0, \theta_{1}^{\prime}(0)=\theta_{1}(\infty)=0 .
$$

The second-order problems are

$$
\left.\begin{array}{l}
f_{2}^{\prime \prime}\left(\eta, c_{1}, c_{2}\right)=\left(1+c_{1}\right) f_{1}^{\prime \prime}+c_{1}\left(\frac{1}{2} f_{0} f_{1}^{\prime \prime}+\frac{1}{2} f_{1} f_{0}^{\prime \prime}+\operatorname{Gr} \theta_{1}-M f_{1}^{\prime}\right)+c_{2}\left(\frac{1}{2} f_{0} f_{0}^{\prime \prime}+\operatorname{Gr} \theta_{0}-M f_{0}^{\prime}\right) \\
\theta_{2}^{\prime \prime}\left(\eta, c_{4}, c_{5}\right)=\left(1+c_{4}\right) \theta_{1}^{\prime \prime}+\frac{1}{2} k_{0} \operatorname{Pr}\left[\left(c_{4} \theta_{1}^{\prime}+c_{5} \theta_{0}^{\prime}\right) f_{0}+c_{4} \theta_{0}^{\prime} f_{1}\right]+\operatorname{Pr} \lambda k_{0}\left(c_{4} \theta_{1}+c_{5} \theta_{0}\right)
\end{array}\right\}
$$

subjected to boundary conditions

$$
f_{2}(0)=f_{2}^{\prime}(0)=f_{2}^{\prime}(\infty)=0, \theta_{3}^{\prime}(0)=\theta_{2}(\infty)=0 .
$$

$$
\left.\begin{array}{c}
f_{3}^{\prime \prime}\left(\eta, c_{1}, c_{2}, c_{3}\right)=\left(1+c_{1}\right) f_{2}^{\prime \prime}+c_{2} f_{1}^{\prime \prime}+\frac{1}{2} c_{1}\left(f_{1} f_{1}^{\prime \prime}+f_{0} f_{2}^{\prime \prime}+f_{0}^{\prime \prime} f_{2}\right)+\frac{1}{2} c_{2}\left(f_{0} f_{1}^{\prime \prime}+f_{0}^{\prime \prime} f_{1}\right) \\
+\frac{1}{2} c_{3} f_{0} f_{0}^{\prime \prime}+\operatorname{Gr}\left(c_{1} \theta_{2}+c_{2} \theta_{1}+c_{3} \theta_{0}\right)+c_{3} f_{0}^{\prime \prime}-M\left(\left(c_{1} f_{2}^{\prime}+c_{2} f_{1}^{\prime}+c_{3} f_{0}^{\prime}\right)\right. \\
\theta_{3}^{\prime \prime}\left(\eta, c_{4}, c_{5}, c_{6}\right)=\left(1+c_{4}\right) \theta_{2}^{\prime \prime}+c_{5} \theta_{1}^{\prime \prime}+\frac{1}{2} \operatorname{Pr} k_{0}\left[\left(c_{4} \theta_{2}^{\prime}+c_{5} \theta_{1}^{\prime}+c_{6} \theta_{0}^{\prime}\right) f_{0}+\left(c_{4} \theta_{1}^{\prime}+c_{5} \theta_{0}^{\prime}\right) f_{1}+c_{4} \theta_{0}^{\prime} f_{2}\right] \\
+\operatorname{Pr} \lambda k_{0}\left(c_{4} \theta_{2}+c_{5} \theta_{1}+c_{6} \theta_{0}\right)
\end{array}\right\},
$$

subjected to boundary conditions

$$
\begin{aligned}
f_{3}(0) & =f_{3}^{\prime}(0)=f_{3}^{\prime}(\infty)=0, \\
\theta_{3}^{\prime}(0) & =\theta_{3}(\infty)=0 .
\end{aligned}
$$

Solving in-sequence equations (44), (46), (48), and (50) with boundary condition equations (45), (47), (49), and (51) and using the OHAM for $p=1$, we obtain the following solutions:

$$
\begin{aligned}
& f_{0}(\eta)=\frac{1}{2 n} \eta^{2}, \\
& \theta_{0}(\eta)=\operatorname{Bi}(1-\theta(0))(n-\eta),
\end{aligned}
$$




$$
\begin{aligned}
& \left\{\begin{array}{l}
f_{1}\left(\eta, c_{1}\right)=c_{1}\left[\frac{1}{48}\left(\frac{\eta^{5}}{5 n^{2}}-\frac{n \eta^{2}}{2}\right)+\frac{G r n B i}{6}(1-\theta(0))\left(\eta^{3}-\frac{\eta^{4}}{4 n}-n \eta^{2}\right)-\frac{M}{12}\left(\frac{\eta^{4}}{2 n}-n \eta^{2}\right)\right] \\
\theta_{1}\left(\eta, c_{4}\right)=c_{4} \operatorname{Prn}{ }^{3} B i(1-\theta(0))\left(\frac{-k_{0}}{48}\left(\frac{\eta^{4}}{n^{4}}-1\right)+k_{0} \lambda\left(\frac{\eta^{2}}{2 n^{2}}-\frac{\eta^{3}}{6 n^{3}}-\frac{1}{3}\right)\right)
\end{array}\right. \\
& f_{2}\left(\eta, c_{1}, c_{2}\right)=\left(1+c_{1}\right) c_{1}\left[\frac{1}{48}\left(\frac{\eta^{5}}{5 n^{2}}+\frac{n \eta^{2}}{2}\right)+\operatorname{GrnBi}(1-\theta(0))\right]\left(\frac{\eta^{3}}{6}-\frac{\eta^{4}}{24 n}-\frac{n \eta^{2}}{6}\right)-\frac{M}{12}\left(\frac{\eta^{4}}{24 n}-n \eta^{2}\right) \\
& +c_{1}\left(\frac{c_{1}}{24}\left(\frac{1}{8}\left(\frac{\eta^{8}}{84 n^{3}}-\frac{\eta^{5}}{60}-\frac{n^{3} \eta^{2}}{168}\right)+\operatorname{GrBi}(1-\theta(0))\left(\frac{\eta^{6}}{20}-\frac{\eta^{7}}{70 n}-\frac{n \eta^{5}}{30}-\frac{n^{4} \eta^{2}}{60}\right)-M\left(\frac{\eta^{7}}{70 n^{2}}-\frac{\eta^{5}}{60}-\frac{n^{3} \eta^{2}}{120}\right)\right)\right. \\
& +\frac{1}{12} c_{1}\left(\frac{1}{8}\left(\frac{\eta^{8}}{1680 n^{3}}-\frac{\eta^{5}}{120}+\frac{31 n^{3} \eta^{2}}{1680}\right)+\operatorname{GrBi}(1-\theta(0))\left(\frac{\eta^{6}}{120}-\frac{\eta^{7}}{840 n}-\frac{n \eta^{5}}{60}+\frac{n^{4} \eta^{2}}{48}\right)-\frac{M}{2}\left(\frac{\eta^{7}}{420 n^{2}}-\frac{\eta^{5}}{60}+\frac{n^{3} \eta^{2}}{30}\right)\right) \\
& +\operatorname{Grc}_{4} \operatorname{prBi}(1-\theta(0))\left[\frac{k_{0}}{48}\left(\frac{n^{3} \eta^{3}}{6}-\frac{\eta^{7}}{210 n}-\frac{7 n^{4} \eta^{2}}{30}\right)+\lambda\left(\frac{n \eta^{5}}{120}-\frac{\eta^{6}}{720}-\frac{n^{3} \eta^{3}}{18}+\frac{n^{4} \eta^{2}}{15}\right)\right] \\
& \left.\cdot \frac{-M c_{1}}{6}\left(\frac{1}{8}\left(\frac{\eta^{7}}{210 n^{2}}-\frac{n \eta^{4}}{24}+\frac{n^{3} \eta^{2}}{15}\right)+\operatorname{GrBi}(1-\theta(0))\left(\frac{n \eta^{5}}{20}-\frac{\eta^{6}}{120}-\frac{n^{2} \eta^{4}}{12}+\frac{n^{4} \eta^{2}}{15}\right)-M\left(\frac{\eta^{6}}{120 n}-\frac{n \eta^{4}}{24}+\frac{7 n^{3} \eta^{2}}{120}\right)\right)\right) \\
& +c_{2}\left(\frac{1}{48}\left(\frac{\eta^{5}}{5 n^{2}}-\frac{n \eta^{2}}{2}\right)+G r n B i(1-\theta(0))\left(\frac{\eta^{3}}{6}-\frac{\eta^{4}}{24 n}-\frac{n \eta^{2}}{6}\right)-\frac{M}{6}\left(\frac{\eta^{4}}{4 n}-\frac{n \eta^{2}}{2}\right)\right) \\
& \theta_{2}\left(\eta, c_{4}, c_{5}\right)=\operatorname{prn}^{3} B i(1-\theta(0))\left[\left(1+c_{4}\right) c_{4}\left[\frac{k_{0}}{48}\left(1-\frac{\eta^{4}}{n^{4}}\right)+\lambda\left(\frac{\eta^{2}}{2 n^{2}}-\frac{\eta^{3}}{6 n^{3}}-\frac{1}{3}\right)\right]+\frac{1}{4} k_{0}\right. \\
& \cdot\left(c_{4}^{2} \operatorname{Pr} n^{2}\left(\frac{k_{0}}{504}\left(1-\frac{\eta^{7}}{n^{7}}\right)+\lambda\left(\frac{\eta^{5}}{20 n^{5}}-\frac{\eta^{6}}{60 n^{6}}-\frac{1}{30}\right)\right)-\frac{c_{5}}{12}\left(1-\frac{\eta^{4}}{n^{4}}\right)\right. \\
& \left.-c_{1} c_{4} n^{2}\left[\frac{1}{24}\left(\frac{\eta^{7}}{210 n^{7}}-\frac{\eta^{4}}{24 n^{4}}+\frac{31}{840}\right)+\frac{G r n B i(1-\theta(0))}{3}\left(\frac{\eta^{5}}{20 n^{5}}-\frac{\eta^{6}}{120 n^{6}}-\frac{\eta^{4}}{12 n^{4}}+\frac{5}{120}\right)-\frac{M}{6}\left(\frac{\eta^{6}}{60 n^{6}}-\frac{\eta^{4}}{12 n^{4}}+\frac{1}{15}\right)\right]\right) \\
& +\lambda\left[c_{5}\left(\frac{\eta^{2}}{2 n^{2}}-\frac{\eta^{3}}{6 n^{3}}-\frac{1}{3}\right)+c_{4}^{2} \operatorname{Prn}^{2}\left(\frac{k_{0}}{48}\left(\frac{\eta^{2}}{2 n^{2}}-\frac{\eta^{6}}{30 n^{6}}-\frac{7}{15}\right)+\lambda\left(\frac{\eta^{4}}{24 n^{4}}-\frac{\eta^{5}}{120 n^{5}}-\frac{\eta^{2}}{6 n^{2}}+\frac{2}{15}\right)\right)\right], \\
& f_{3}=\left(1+c_{1}\right)\left\{\left(1+c_{1}\right) c_{1}\left(\frac{1}{240 n^{2}}\left(\eta^{5}-\eta^{2}\right)+\frac{1}{6} B i[1-\theta(0)] G r n\left(\eta^{3}-\eta^{2}-\frac{1}{4 n}\left(\eta^{4}-\eta^{2}\right)\right)-\frac{M}{24 n}\left(\eta^{4}-\eta^{2}\right)\right)\right. \\
& +c_{1}\left(\frac { 1 } { 2 4 } c _ { 1 } \left(\frac{1}{672 n^{3}}\left(\eta^{8}-\eta^{2}\right)+\frac{1}{480} \eta^{5}+\frac{1}{480} \eta^{2}+\operatorname{BiGr}[1-\theta(0)]\left(\frac{1}{20} \eta^{6}-\frac{1}{20} \eta^{2}-\frac{1}{70 n}\left(\eta^{7}-\eta^{2}\right)\right.\right.\right. \\
& -\frac{1}{30}\left(\eta^{5}-\eta^{2}\right) n-M\left(\frac{1}{70 n^{2}}\left(\eta^{7}-\eta^{2}\right)+\frac{1}{60}\left(\eta^{2}-\eta^{5}\right)\right)+\frac{1}{12} c_{1}\left(\frac{1}{13440 n^{3}}\left(\eta^{8}-\eta^{2}\right)+\frac{1}{960}\left(\eta^{2}-\eta^{5}\right)\right. \\
& +\operatorname{BiGr}[1-\theta(0)] \frac{1}{120}\left(\eta^{6}-\eta^{2}\right)-\frac{1}{840 n}\left(\eta^{7}-\eta^{2}\right)-\frac{1}{60}\left(\eta^{5}-\eta^{2}\right) n-\frac{M}{2}\left(\frac{1}{420 n^{2}}\left(\eta^{7}-\eta^{2}\right)-\frac{1}{60}\left(\eta^{5}-\eta^{2}\right)\right) \\
& +G r \operatorname{Pr} B i[1-\theta(0)] c 4\left(\frac{1}{48} k_{0}\left(\frac{1}{6} n^{3}\left(\eta^{3}-\eta^{2}\right)-\frac{1}{210 n}\left(\eta^{7}-\eta^{2}\right)\right)+\lambda\left(\frac{1}{120} n\left(\eta^{5}-\eta^{2}\right)-\frac{1}{720}\left(\eta^{6}-\eta^{2}\right)-\frac{1}{18}\left(\eta^{3}-\eta^{2}\right) n^{3}\right)\right) \\
& -\frac{1}{6} M c_{1}\left(\frac{1}{680 n^{2}}\left(\eta^{7}-\eta^{2}\right)-\frac{1}{192}\left(\eta^{4}-\eta^{2}\right) n+\operatorname{BiGr}[1-\theta(0)] n\left(\frac{1}{20}\left(\eta^{5}-\eta^{2}-\frac{1}{6 n}\left(\eta^{6}-\eta^{2}\right)\right)\right.\right. \\
& \left.\left.-\frac{1}{12}\left(\eta^{4}-\eta^{2}\right) n-M\left(\frac{1}{120 n}\left(\eta^{6}-\eta^{2}\right)-\frac{n}{24}\left(\eta^{4}-\eta^{2}\right)\right)\right)\right) \\
& \left.+c_{2}\left(\frac{1}{240 n^{2}}\left(\eta^{5}-\eta^{2}\right) \operatorname{BiGrn}[1-\theta(0)]\left(\frac{1}{6} \eta^{3}-\frac{1}{6} \eta^{2}-\frac{1}{24 n}\left(\eta^{4}-\eta^{2}\right)\right)-\frac{M}{24 n}\left(\eta^{4}-\eta^{2}\right)\right)\right\} \\
& +c_{2} c_{1}\left(\frac{1}{240 n^{2}}\left(\eta^{5}-\eta^{2}\right)+\frac{1}{6} \operatorname{BiGrn}[1-\theta(0)]\left(\eta^{4}-\eta^{2}-\frac{1}{4 n}\left(\eta^{4}-\eta^{2}\right)\right)-\frac{M}{24 n}\left(\eta^{4}-\eta^{2}\right)\right)
\end{aligned}
$$




$$
\begin{aligned}
& \frac{1}{2} c_{1}^{3}\left(\frac{3.5073 \times 10^{-7}}{n^{4}}\left(\eta^{11}-\eta^{2}\right)+\left(\frac{1}{720 n^{2}}\left(\frac{-1}{288} G r B i[1-\theta(0)] \frac{-M}{288 n}\right)+\frac{1}{172800 n^{2}}\left(\frac{-1}{2}\left(\operatorname{BiGr}[1-\theta(0)]-\frac{M}{n}\right)\right)\right)\left(\eta^{10}-\eta^{2}\right)\right) \\
& +\left(\frac{13}{362880 n} \operatorname{BiGr}[1-\theta(0)]+\frac{1}{504}\left(\frac{-1}{24}\left(\operatorname{BiGr}[1-\theta(0)]+\frac{M}{n}\right)\right)\left(\frac{-1}{2}\left(\operatorname{BiGr}[1-\theta(0)]+\frac{M}{n}\right)\right)\right)\left(\eta^{9}-\eta^{2}\right) \\
& +\frac{1}{336}\left(\frac{-1}{1152} n-\frac{1}{72} \operatorname{BiGrn}^{2}[1-\theta(0)]+\frac{M n}{144 n^{2}}+\frac{\operatorname{BiGr}[1-\theta(0)] n}{2016}\left(\frac{\operatorname{BiGr}[1-\theta(0)] n}{2}-\frac{M}{2 n}\right)\right. \\
& \left.+\frac{B i G r n[1-\theta(0)] n}{336}\left(\frac{-B i G r[1-\theta(0)] n}{24}-\frac{M}{24 n}\right)+\frac{1}{80640 n^{2}}\left(\frac{-n}{48}-\frac{B i G r[1-\theta(0)]}{3} n^{2}+\frac{M n}{6}\right)\right)\left(\eta^{8}-\eta^{2}\right) \\
& \left.+\left(\frac{1}{210}\left(\frac{-n}{96}-\frac{B i G r[1-\theta(0)]}{6} n^{2}+\frac{M n}{12}\right)\left(\frac{-B i G r[1-\theta(0)]}{2}-\frac{M}{2 n}\right)+\frac{1}{1260}(\operatorname{Gr} \operatorname{Bin}[1-\theta(0)]]\right)^{2}\right) \\
& \left.+\frac{1}{210}\left(\frac{-B i G r[1-\theta(0)]}{24}-\frac{M}{24 n}\right)\left(\frac{-n}{48}-\frac{B i G r[1-\theta(0)]}{3} n^{2}+\frac{M n}{6}\right)\right)\left(\eta^{7}-\eta^{2}\right) \\
& +\left(\frac{B i G r n[1-\theta(0)] n}{120}\left(\frac{-n}{96}-\frac{B i G r n[1-\theta(0)]}{6} n^{2}+\frac{M n}{12}\right) \frac{\operatorname{BiGrn}[1-\theta(0)]}{720}\left(\frac{-n}{48}-\frac{B i G r n[1-\theta(0)]}{3} n^{2}+\frac{M n}{6}\right)\right)\left(\eta^{6}-\eta^{2}\right) \\
& +\left(\frac{-n}{5760}-\frac{B i G r n[1-\theta(0)]}{360} n^{2}+\frac{M n}{720}\right)\left(\frac{-n}{48}-\frac{B i G r n[1-\theta(0)]}{3} n^{2}+\frac{M n}{6}\right)\left(\eta^{5}-\eta^{2}\right) \\
& +\frac{c_{1} c_{2} G r}{4 n}\left(c _ { 1 } c _ { 2 } \left(\frac{n}{10080 n^{2}}\left(\eta^{7}-\eta^{2}\right)-\frac{n}{1152}\left(\eta^{4}-\eta^{2}\right)+\operatorname{BiGrn}[1-\theta(0)]\left(\frac{1}{20 n}\left(\eta^{5}-\eta^{2}\right)-\frac{n}{12}\left(\eta^{4}-\eta^{2}\right)\right)\right.\right. \\
& \left.\left.-\frac{M}{12}\left(\frac{1}{60 n}\left(\eta^{6}-\eta^{2}\right)-\frac{n}{12}\left(\eta^{4}-\eta^{2}\right)\right)\right)+\frac{c_{3}}{24 n}\left(\eta^{4}-\eta^{2}\right)\right) \\
& +\frac{1}{4 n} c_{1}\left(\frac{3.8580 \times 10^{-6}}{n^{3}} c_{1}^{2}\left(\eta^{11}-\eta^{2}\right)+\frac{c_{1}}{720}\left(\frac{1}{12} c_{1}\left(-\frac{B i G r[1-\theta(0)]}{20 n}-\frac{M}{20 n^{2}}\right)\right.\right. \\
& \left.+\frac{1}{24} c_{1}\left(\frac{-3 B i G r[1-\theta(0)]}{5 n}-\frac{3 M}{5 n^{2}}\right) \frac{-G r \operatorname{Pr} B i k_{0} c_{4[1-\theta(0)]}}{240 n}-\frac{M c_{1}}{240 n^{2}}\right)\left(\eta^{10}-\eta^{2}\right) \\
& +\frac{1}{504} c_{1}\left(\frac{-G r \operatorname{Pr} B i \lambda c_{4[1-\theta(0)]}}{24}+\frac{G r B i c_{1}[1-\theta(0)]}{12}-\frac{M c_{1}}{6}\left(\frac{-G r B i[1-\theta(0)]}{4}-\frac{M}{4 n}\right)\right)\left(\eta^{9}-\eta^{2}\right) \\
& \cdot\left(\frac{c_{1}}{336 n^{2}}\left(\frac{1}{12}+\frac{c_{1}}{12}\right)+\frac{c_{2}}{4032 n^{2}}+\frac{c_{1}}{336}\left(\frac{-G r M B i n c_{1}[1-\theta(0)]}{6}+\frac{c_{1}}{24}\left(\frac{-2 G r B i n[1-\theta(0)]}{3}-\frac{1}{24}+\frac{M}{3}\right)\right.\right. \\
& \left.\left.+\frac{G r \operatorname{Pr} B i \lambda c_{4}[1-\theta(0)]}{6} n+\frac{c_{1}}{12}\left(\frac{-G r B i n[1-\theta(0)]}{3}-\frac{1}{48}+\frac{M}{6}\right)\right)\right)\left(\eta^{8}-\eta^{2}\right) \\
& +\left(\frac{c_{1}\left(1+c_{1}\right)}{210}\left(\frac{-B i G r[1-\theta(0)]}{2}-\frac{M}{2 n}\right)-\frac{c_{1}^{2} M}{1260}\left(-\operatorname{BiGr}[1-\theta(0)] n^{2}-\frac{n}{16}+\frac{M n}{2}\right)+\frac{c_{2}}{210}\left(\frac{-B i G r[1-\theta(0)]}{2}-\frac{M}{2 n}\right)\right)\left(\eta^{7}-\eta^{2}\right) \\
& +\left(\frac{c_{1}\left(1+c_{1}\right)}{120} \operatorname{BiGr}[1-\theta(0)] n+\frac{\operatorname{Pr} B i G r[1-\theta(0)]}{120} c_{1} c_{4}\left(\frac{k_{0} n^{3}}{48}-\frac{\lambda n^{3}}{3}\right)+\frac{c_{2} B i G r n[1-\theta(0)]}{120}\right)\left(\eta^{6}-\eta^{2}\right) \\
& +\left(\frac{c_{1}\left(1+c_{1}\right)}{60}\left(\frac{-n}{48}-\frac{B i G r n^{2}[1-\theta(0)]}{3}+\frac{M n}{6}\right)+\frac{c_{2}}{60}\left(\frac{-n}{48}-\frac{B i G r n^{2}[1-\theta(0)]}{3}+\frac{M n}{6}\right)\right. \\
& +\frac{c_{1}}{60}\left(\frac{c_{1}}{24}\left(\frac{-n^{3}}{672}-\frac{B i G r n^{4}[1-\theta(0)]}{30}+\frac{M n^{3}}{60}\right)+\frac{c_{1}}{12}\left(\frac{31 n^{3}}{6720}+\frac{B i G r n^{4}[1-\theta(0)]}{24}-\frac{M n^{3}}{30}\right)\right. \\
& \left.\left.\left.\cdot \frac{-M c_{1}}{6}\left(\frac{n^{3}}{60}+\frac{2 B i G r n^{4}[1-\theta(0)]}{15}-\frac{7 M n^{3}}{60}\right)+G r c_{4} \operatorname{Pr} B i[1-\theta(0)]\left(\frac{-k_{0} n^{4}}{720}+\frac{2 \lambda n^{4}}{15}\right)\right)\right)\right)\left(\eta^{5}-\eta^{2}\right)
\end{aligned}
$$


International Journal of Differential Equations

9

$$
\begin{aligned}
& +\frac{1}{2 n} c_{1}^{2}\left(c_{1}+1\right)\left(\frac{1}{80640 n^{2}}\right)\left(\eta^{8}-\eta^{2}\right)-\frac{n}{5760}\left(\eta^{5}-\eta^{2}\right) \\
& +\frac{B i G r n[1-\theta(0)]}{6}\left(\frac{1}{120}\left(\eta^{6}-\eta^{2}\right)-\frac{1}{480 n}\left(\eta^{7}-\eta^{2}\right)-\frac{n \eta^{5}}{6}\right) \frac{-M}{12}\left(\frac{1}{420 n}\left(\eta^{7}-\eta^{2}\right)-\frac{n}{60}\left(\eta^{5}-\eta^{2}\right)\right) \\
& +\frac{c_{1}^{3}}{48 n}-\left(\frac{M c_{1}}{6}\left(\frac{8.2672 \times 10^{-7}}{n^{2}}\right)\left(\eta^{10}-\eta^{2}\right)-\frac{n}{40320}\left(\eta^{7}-\eta^{2}\right)+\frac{n^{3}}{7200}\left(\eta^{5}-\eta^{2}\right)+\operatorname{BiGrn}[1-\theta(0)]\right. \\
& \cdot\left(\frac{1}{6720}\left(\eta^{8}-\eta^{2}\right)-\frac{1}{60480 n}\left(\eta^{9}-\eta^{2}\right)-\frac{n}{2520}\left(\eta^{7}-\eta^{2}\right)+\frac{n^{3}}{900}\left(\eta^{5}-\eta^{2}\right)\right) \\
& -M\left(\frac{1}{60480 n}\left(\eta^{9}-\eta^{2}\right)-\frac{n}{5040}\left(\eta^{7}-\eta^{2}\right)-\frac{1}{5040}\left(\frac{7 n^{2}}{7200}\left(\eta^{5}-\eta^{2}\right)\right)\right) \\
& +c_{2}\left(\frac{1}{80640 n^{2}}\left(\eta^{8}-\eta^{2}\right)-\frac{n}{5760}\left(\eta^{5}-\eta^{2}\right)+\operatorname{BiGrn}[1-\theta(0)]\left(\frac{1}{720}\left(\eta^{6}-\eta^{2}\right)-\frac{1}{5040 n}\left(\eta^{7}-\eta^{2}\right)-\frac{n}{360}\left(\eta^{5}-\eta^{2}\right)\right)\right. \\
& \left.\left.-\frac{M}{6}\left(\frac{1}{840 n}\left(\eta^{7}-\eta^{2}\right)-\frac{n}{120}\left(\eta^{5}-\eta^{2}\right)\right)\right)\right) \\
& +\frac{c_{1} c_{2}}{4 n}\left(\frac{c_{3}}{240 n^{2}}\left(\eta^{5}-\eta^{2}\right) G r \operatorname{Pr} B i c_{1}[1-\theta(0)] n^{3}\right)\left(c _ { 4 } ( 1 + c _ { 4 } ) \left(\lambda\left(\frac{1}{120 n^{2}}\left(\eta^{5}-\eta^{2}\right)-\frac{1}{720 n^{3}}\left(\eta^{6}-\eta^{2}\right)-\frac{1}{18}\left(\eta^{3}-\eta^{2}\right)\right)\right.\right. \\
& \left.-\frac{k_{0}}{48}\left(\frac{1}{210 n^{4}}\left(\eta^{7}-\eta^{2}\right)-\frac{1}{6}\left(\eta^{3}-\eta^{2}\right)\right)\right) \\
& +\frac{k_{0}}{4}\left(c_{4}^{2} n^{2} \operatorname{Pr}\left(\lambda\left(\frac{1}{6720 n^{5}}\left(\eta^{8}-\eta^{2}\right)-\frac{1}{30240 n^{6}}\left(\eta^{9}-\eta^{2}\right)-\frac{1}{180}\left(\eta^{3}-\eta^{2}\right)\right)+\frac{k_{0}}{504}\left(\frac{1}{6}\left(\eta^{3}-\eta^{2}\right)-\frac{1}{720 n^{7}}\left(\eta^{10}-\eta^{2}\right)\right)\right)\right. \\
& \left.\left.+\frac{c_{5}}{72}\left(\eta^{3}-\eta^{2}\right)-\frac{1}{210 n^{4}}\left(\eta^{7}-\eta^{2}\right)\right)\right) \\
& -M \frac{c_{1}^{2} c_{2} G r}{4 n}\left(1+c_{1}\right)\left(\frac{1}{10080 n^{2}}\left(\eta^{7}-\eta^{2}\right)-\frac{n}{1152}\left(\eta^{4}-\eta^{2}\right)+\frac{\operatorname{BiGrn}[1-\theta(0)]}{6}\left(\frac{1}{20}\left(\eta^{5}-\eta^{2}\right)\right.\right. \\
& \left.\left.-\frac{1}{120 n}\left(\eta^{6}-\eta^{2}\right)-\frac{n}{12}\left(\eta^{4}-\eta^{2}\right)\right) \frac{-M}{12}\left(\frac{1}{60 n}\left(\eta^{6}-\eta^{2}\right)-\frac{n}{12}\left(\eta^{4}-\eta^{2}\right)\right)\right) \\
& +\frac{c_{3}^{1}}{48 n}\left(\frac{1.5031 \times 10^{-6}}{n^{3}}\left(\eta^{11}-\eta^{2}\right)-6.2004 \times 10^{-6}\left(\eta^{8}-\eta^{2}\right)-\frac{n^{3}}{80640}\left(\eta^{5}-\eta^{2}\right)\right. \\
& +\operatorname{BiGr}[1-\theta(0)]\left(\frac{1}{10080}\left(\eta^{9}-\eta^{2}\right)-\frac{1}{50400 n}\left(\eta^{10}-\eta^{2}\right)-\frac{n}{10080}\left(\eta^{8}-\eta^{2}\right)-\frac{n^{4}}{3600}\left(\eta^{5}-\eta^{2}\right)\right) \\
& -M\left(\frac{1}{50400 n^{2}}-\frac{1}{20160}\left(\eta^{8}-\eta^{2}\right)-\frac{n^{3}}{7200}\left(\eta^{5}-\eta^{2}\right)\right) \\
& +\frac{c_{1}}{12}\left(\frac{7.5156 \times 10^{-8}}{n^{3}}\left(\eta^{11}-\eta^{2}\right)-3.1002 \times 10^{-6}\left(\eta^{8}-\eta^{2}\right)+3.8442 \times 10^{-5} n^{3}\left(\eta^{5}-\eta^{2}\right)\right. \\
& +\operatorname{BiGr}[1-\theta(0)]\left(\frac{1}{60480}\left(\eta^{9}-\eta^{2}\right)-\frac{1.6534 \times 10^{-6}}{n}\left(\eta^{10}-\eta^{2}\right)-\frac{n}{20160}\left(\eta^{8}-\eta^{2}\right)+\frac{n^{4}}{2880}\left(\eta^{5}-\eta^{2}\right)\right) \\
& -\frac{M}{2}\left(\frac{3.3069 \times 10^{-6}}{n^{2}}\left(\eta^{10}-\eta^{2}\right)-\frac{1}{20160}\left(\eta^{8}-\eta^{2}\right)+\frac{n^{3}}{1800}\left(\eta^{5}-\eta^{2}\right)\right)
\end{aligned}
$$




$$
\begin{aligned}
& \cdot \operatorname{BiPrGrc}_{4}[1-\theta(0)]\left(\frac{k_{0}}{48}\left(\frac{n^{3}}{720}\left(\eta^{5}-\eta^{2}\right)-\frac{6.6138 \times 10^{-6}}{n}\left(\eta^{10}-\eta^{2}\right)-\frac{n^{4}}{1800}\left(\eta^{5}-\eta^{2}\right)\right)\right. \\
& \left.\left.+\lambda\left(\frac{n}{40320}\left(\eta^{8}-\eta^{2}\right)-2.7557 \times 10^{-6}\left(\eta^{9}-\eta^{2}\right)-\frac{n^{3}}{2160}\left(\eta^{6}-\eta^{2}\right)+\frac{n^{4}}{900}\left(\eta^{5}-\eta^{2}\right)\right)\right)\right) \\
& -M \frac{c_{1}^{2} c_{2} G r}{4 n}\left(\frac { c _ { 1 } } { 2 4 } \left(\frac{1}{60480 n^{3}}\left(\eta^{10}-\eta^{2}\right)-\frac{1}{20160}\left(\eta^{7}-\eta^{2}\right)-\frac{n^{3}}{16128}\left(\eta^{4}-\eta^{2}\right)+B i G r[1-\theta(0)]\right.\right. \\
& \cdot\left(\frac{1}{1120}\left(\eta^{8}-\eta^{2}\right)-\frac{1}{5040 n}\left(\eta^{9}-\eta^{2}\right)-\frac{n}{1260}\left(\eta^{7}-\eta^{2}\right)-\frac{n^{4}}{720}\left(\eta^{4}-\eta^{2}\right)\right) \\
& \left.-M\left(\frac{1}{5040 n^{2}}\left(\eta^{9}-\eta^{2}\right)-\frac{1}{2520}\left(\eta^{7}-\eta^{2}\right)-\frac{n^{3}}{1440}\left(\eta^{4}-\eta^{2}\right)\right)\right) \\
& +\frac{c_{1}}{12}\left(\frac{8.2672 \times 10^{-7}}{n^{3}}\left(\eta^{10}-\eta^{2}\right)-\frac{1}{40320}\left(\eta^{7}-\eta^{2}\right)+1.9221 \times 10^{-4} n^{3}\left(\eta^{4}-\eta^{2}\right)\right. \\
& +B i G r[1-\theta(0)]\left(\frac{1}{6720}\left(\eta^{8}-\eta^{2}\right)-\frac{1}{60480 n}\left(\eta^{9}-\eta^{2}\right)-\frac{n}{2520}\left(\eta^{7}-\eta^{2}\right)+\frac{n^{4}}{576}\left(\eta^{4}-\eta^{2}\right)\right) \\
& \left.\frac{-M}{2}\left(\frac{1}{30240 n^{2}}\left(\eta^{9}-\eta^{2}\right)-\frac{1}{2520}\left(\eta^{7}-\eta^{2}\right)+\frac{n^{3}}{360}\left(\eta^{4}-\eta^{2}\right)\right)\right) \\
& +\operatorname{PrBiGrc_{4}}[1-\theta(0)]\left(\frac{k_{0}}{48}\left(\frac{n^{3}}{120}\left(\eta^{5}-\eta^{2}\right)-\frac{1}{15120 n}\left(\eta^{9}-\eta^{2}\right)-\frac{n^{4}}{360}\left(\eta^{4}-\eta^{2}\right)\right)\right. \\
& \left.+\lambda\left(\frac{n}{5040}\left(\eta^{7}-\eta^{2}\right)-\frac{1}{40320}\left(\eta^{8}-\eta^{2}\right)-\frac{n^{3}}{360}\left(\eta^{5}-\eta^{2}\right)+\frac{n^{4}}{180}\left(\eta^{4}-\eta^{2}\right)\right)\right) \\
& \frac{-M c_{1}}{6}\left(\frac{8.2672 \times 10^{-6}}{n^{2}}\left(\eta^{9}-\eta^{2}\right)-\frac{n}{5760}\left(\eta^{6}-\eta^{2}\right)+\frac{n^{3}}{1440}\left(\eta^{4}-\eta^{2}\right)\right. \\
& +\operatorname{BiGrn}[1-\theta(0)]\left(\frac{1}{840}\left(\eta^{7}-\eta^{2}\right)-\frac{1}{6720 n}\left(\eta^{8}-\eta^{2}\right)-\frac{n}{360}\left(\eta^{6}-\eta^{2}\right)+\frac{n^{3}}{180}\left(\eta^{4}-\eta^{2}\right)\right) \\
& \left.\left.-M\left(\frac{1}{6720 n}\left(\eta^{8}-\eta^{2}\right)-\frac{n}{720}\left(\eta^{6}-\eta^{2}\right)+\frac{7 n^{3}}{1440}\left(\eta^{4}-\eta^{2}\right)\right)\right)\right) \\
& -\frac{M c_{1}^{2} c_{2} G r}{4 n}\left(\frac{1}{10080 n^{2}}\left(\eta^{7}-\eta^{2}\right)-\frac{n}{1152}\left(\eta^{4}-\eta^{2}\right)+B i G r n[1-\theta(0)]\left(\frac{1}{120 n}\left(\eta^{5}-\eta^{2}\right)\right.\right. \\
& \left.\left.-\frac{1}{720 n}\left(\eta^{6}-\eta^{2}\right)-\frac{n}{72}\left(\eta^{4}-\eta^{2}\right)\right)-\frac{M}{6}\left(\frac{1}{120 n}\left(\eta^{6}-\eta^{2}\right)-\frac{n}{24}\left(\eta^{4}-\eta^{2}\right)\right)\right) \\
& +\frac{c_{1}^{2} c_{2}}{4 n}\left(\frac{1}{4032 n^{2}}\left(\eta^{8}-\eta^{2}\right)-\frac{1}{420}(B i G r[1-\theta(0)])+\frac{M}{n}\left(\eta^{7}-\eta^{2}\right)+\frac{B i G r[1-\theta(0)] n}{120}\right)\left(\eta^{6}-\eta^{2}\right) \\
& +\left(\frac{-n}{2880}-\frac{B i G r[1-\theta(0)] n^{2}}{180}+\frac{M n}{360}\right)\left(\eta^{5}-\eta^{2}\right) \\
& +\frac{c_{1}}{n}\left(\frac{1}{80640 n^{2}}\left(\eta^{7}-\eta^{2}\right)-\frac{n}{5760}\left(\eta^{4}-\eta^{2}\right)+\frac{B i G r[1-\theta(0)] n}{6}\left(\frac{1}{120}\left(\eta^{6}-\eta^{2}\right)-\frac{1}{840 n}\left(\eta^{7}-\eta^{2}\right)-\frac{n}{60}\left(\eta^{5}-\eta^{2}\right)\right.\right. \\
& \left.\left.-\frac{M}{12}\left(\frac{1}{420 n}\left(\eta^{7}-\eta^{2}\right)-\frac{n}{60}\left(\eta^{5}-\eta^{2}\right)\right)\right)\right)
\end{aligned}
$$




$$
\begin{aligned}
& +\frac{c_{1} c_{2} n^{3}}{4 n} \operatorname{Gr} \operatorname{Pr} B i c_{1}[1-\theta(0)]\left(\frac { - c _ { 1 } c _ { 4 } n ^ { 2 } c _ { 5 } k _ { 0 } } { 4 8 } \left(\frac{2.7557 \times 10^{-7}}{n^{7}}\left(\eta^{10}-\eta^{2}\right) \frac{-8.2672 \times 10^{-7}}{n^{4}}\left(\eta^{7}-\eta^{2}\right)+2.5628 \times 10^{-4}\left(\eta^{3}-\eta^{2}\right)\right.\right. \\
& +\frac{G r B i[1-\theta(0)] n}{3}\left(\frac{1}{6720 n^{5}}\left(\eta^{8}-\eta^{2}\right)-\frac{1}{60480 n^{6}}\left(\eta^{9}-\eta^{2}\right)-\frac{1}{2520 n^{4}}\left(\eta^{7}-\eta^{2}\right)-\frac{1}{144}\left(\eta^{3}-\eta^{2}\right)\right) \\
& \cdot \frac{-M}{6}\left(\frac{1}{30240 n^{6}}\left(\eta^{9}-\eta^{2}\right)-\frac{1}{2520 n^{4}}\left(\eta^{7}-\eta^{2}\right)-\frac{1}{90}\left(\eta^{3}-\eta^{2}\right)\right) \\
& \cdot \frac{k_{0}}{4} \lambda\left(\frac{c_{5}}{120 n^{2}} \eta^{5}-\frac{1}{720 n^{3}}\left(\eta^{6}-\eta^{2}\right)-\frac{1}{18}\left(\eta^{3}-\eta^{2}\right)\right) \\
& +c_{4}^{2} n^{2} \operatorname{Pr}\left(\begin{array}{c}
\frac{k_{0}}{48}\left(\frac{1}{120 n^{2}}\left(\eta^{5}-\eta^{2}\right)-\frac{1}{15120 n^{6}}\left(\eta^{9}-\eta^{2}\right)-\frac{7}{90}\left(\eta^{3}-\eta^{2}\right)\right) \\
\lambda\left(\frac{1}{5040 n^{4}}\left(\eta^{7}-\eta^{2}\right)-\frac{1}{40320 n^{5}}\left(\eta^{8}-\eta^{2}\right)-\frac{1}{360 n^{2}}\left(\eta^{5}-\eta^{2}\right)+\frac{1}{45}\left(\eta^{3}-\eta^{2}\right)\right)
\end{array}\right)
\end{aligned}
$$

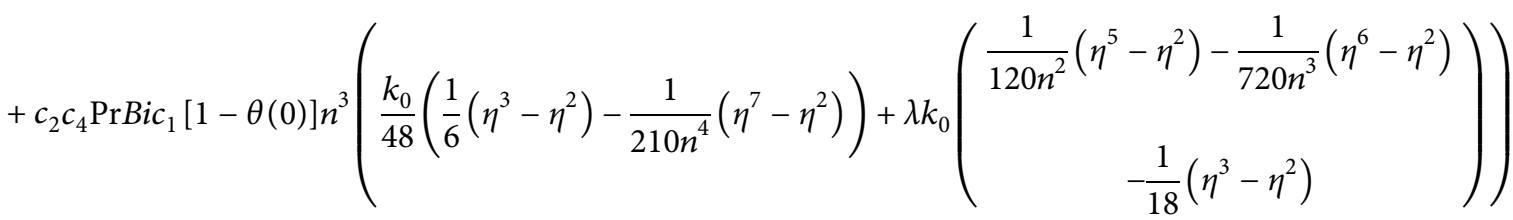

$$
\begin{aligned}
& +c_{3} B i[1-\theta(0)]\left(\frac{n}{6}\left(\eta^{3}-\eta^{2}\right)-\frac{1}{24}\left(\eta^{4}-\eta^{2}\right)\right) \text {. }
\end{aligned}
$$

Adding up solution components (52)-(56), the fourterm solution obtained by the OHAM for $p=1$ is

$$
\left\{\begin{array}{l}
\tilde{f}\left(\eta, c_{1}, c_{2}, c_{3}\right)=f_{0}(\eta)+f_{1}\left(\eta, c_{1}\right)+f_{2}\left(\eta, c_{1}, c_{2}\right)+f_{3}\left(\eta, c_{1}, c_{2}, c_{3}\right), \\
\tilde{\theta}\left(\eta, c_{4}, c_{5}, c_{6}\right)=\theta_{0}(\eta)+\theta_{1}\left(\eta, c_{4}\right)+\theta_{2}\left(\eta, c_{4}, c_{5}\right)+\theta_{3}\left(\eta, c_{4}, c_{5}, c_{6}\right) .
\end{array}\right.
$$

For the calculations of the unknown convergence-control parameters in equation (56), we used the least square method for Prandtl number, $P r=0.72$, Grashof number, $G r=0.1$, radiation parameter, $R a=0.1$, magnetic parameter, $M=0.1$, and internal heat generation, $\lambda=0.1$, and we obtain

$$
\begin{aligned}
& c_{1}=0.16266623, \\
& c_{2}=0.33462218, \\
& c_{3}=0.00016952, \\
& c_{4}=37.96314130, \\
& c_{5}=15845.12896756, \\
& c_{6}=0.0018555 .
\end{aligned}
$$

\section{Results and Discussion}

The system of nonlinear higher-order ordinary differential equation (40) with boundary conditions (41) was solved analytically via the optimal homotopy asymptotic method (OHAM). Analytical computations have been carried out for different embedded parameters in the flow model controlling the fluid dynamics in the flow regime. The influence of different parameters in the flow model on the velocity and temperature profiles has been analyzed for different values of Prandtl number $(P r)$, internal heat generation $(\lambda)$, magnetic parameter $(M)$, Biot number $(B i)$, radiation parameter $(R a)$, and Grashof number $(G r)$, and the results have been displayed in figures and tables for the selected parameters.

Table 1 depicts the validity of the method for the considered boundary layer flow. In Table 1, a comparison of the 
TABLE 1: A comparison of the values obtained for the Nusselt number and surface temperature with an increase in the local Biot number in this study and by Olanrewaju et al., Aziz, Ishak, and Makinde and Oladapo Olanrewaju in previous studies.

\begin{tabular}{|c|c|c|c|c|c|c|c|c|}
\hline \multirow[t]{2}{*}{$B i$} & \multicolumn{2}{|c|}{$\begin{array}{l}\text { OHAM (present } \\
\text { study }\end{array}$} & \multicolumn{2}{|c|}{ Olanrewaju et al. [7] } & \multicolumn{2}{|c|}{ Aziz [22] } & \multirow{2}{*}{$\begin{array}{c}\text { Ishak [20] } \\
-\theta^{\prime}(0) \\
\end{array}$} & \multirow{2}{*}{$\begin{array}{l}\text { Makinde and Oladapo Olanrewaju [24] } \\
\qquad \theta^{\prime}(0)\end{array}$} \\
\hline & $-\theta^{\prime}(0)$ & $\theta(0)$ & $-\theta^{\prime}(0)$ & $\theta(0)$ & $-\theta^{\prime}(0)$ & $\theta(0)$ & & \\
\hline 0.05 & 0.042764 & 0.14464 & 0.042767 & 0.14466 & 0.0428 & 0.1447 & 0.042767 & 0.0428 \\
\hline 0.10 & 0.074722 & 0.25272 & 0.074724 & 0.25275 & 0.0747 & 0.2528 & 0.074724 & 0.0747 \\
\hline 0.20 & 0.119296 & 0.40351 & 0.119295 & 0.40352 & 0.1193 & 0.4035 & 0.119295 & 0.1193 \\
\hline 0.40 & 0.169993 & 0.57501 & 0.169994 & 0.57501 & 0.1700 & 0.5750 & 0.169994 & 0.1700 \\
\hline 0.60 & 0.198049 & 0.66990 & 0.198051 & 0.66991 & 0.1981 & 0.6699 & 0.198051 & 0.1981 \\
\hline 0.80 & 0.215862 & 0.73015 & 0.215864 & 0.73016 & 0.2159 & 0.7302 & 0.215864 & 0.2159 \\
\hline 1.00 & 0.228176 & 0.77179 & 0.228178 & 0.77181 & 0.2282 & 0.7718 & 0.228178 & 0.2282 \\
\hline 5.00 & 0.279129 & 0.94415 & 0.279131 & 0.94417 & 0.2791 & 0.9441 & 0.279131 & 0.2791 \\
\hline 10.00 & 0.287145 & 0.97127 & 0.287146 & 0.97128 & 0.2871 & 0.9713 & 0.287146 & 0.2871 \\
\hline 20.00 & 0.291331 & 0.98541 & 0.291329 & 0.98543 & 0.2913 & 0.9854 & 0.291329 & 0.2913 \\
\hline 30.00 & 0.292752 & 0.99023 & 0.292754 & 0.99024 & - & - & - & 0.2928 \\
\hline
\end{tabular}

TABLE 2: A comparison of values of the skin-friction coefficient and the Nusselt number obtained by the OHAM in the present study and numerical solutions reported by Olanrewaju et al. for various parameter values entrenched in the flow model.

\begin{tabular}{|c|c|c|c|c|c|c|c|c|}
\hline \multirow[t]{2}{*}{$B i$} & \multirow[t]{2}{*}{$G r$} & \multirow[t]{2}{*}{$\operatorname{Pr}$} & \multirow[t]{2}{*}{$\lambda$} & \multirow[t]{2}{*}{$R a$} & \multicolumn{2}{|c|}{ OHAM result } & \multicolumn{2}{|c|}{$\begin{array}{l}\text { Numerical result reported by } \\
\text { Olanrewaju et al. [7] }\end{array}$} \\
\hline & & & & & $f^{\prime \prime}(0)$ & $-\theta^{\prime}(0)$ & $f^{\prime \prime}(0)$ & $-\theta^{\prime}(0)$ \\
\hline 0.1 & 0.1 & 0.72 & 0.1 & 0.1 & 0.386314 & 0.066809 & 0.386316 & 0.066810 \\
\hline 1.0 & 0.1 & 0.72 & 0.1 & 0.1 & 0.460824 & 0.176788 & 0.460825 & 0.176790 \\
\hline 10 & 0.1 & 0.72 & 0.1 & 0.1 & 0.483259 & 0.213879 & 0.483261 & 0.213880 \\
\hline 0.1 & 0.5 & 0.72 & 0.1 & 0.1 & 0.557238 & 0.069728 & 0.557241 & 0.069730 \\
\hline 0.1 & 1.0 & 0.72 & 0.1 & 0.1 & 0.723309 & 0.071735 & 0.723310 & 0.071736 \\
\hline 0.1 & 0.1 & 3.00 & 0.1 & 0.1 & -0.074539 & 0.231310 & -0.074540 & 0.231312 \\
\hline 0.1 & 0.1 & 7.10 & 0.1 & 0.1 & -0.015859 & 0.261731 & -0.015860 & 0.261733 \\
\hline 0.1 & 0.1 & 0.72 & 0.5 & 0.1 & 0.280068 & 0.110630 & 0.280070 & 0.110631 \\
\hline 0.1 & 0.1 & 0.72 & 0.6 & 0.1 & 0.298363 & 0.102050 & 0.298365 & 0.102052 \\
\hline 0.1 & 0.1 & 0.72 & 0.1 & 0.5 & 0.392334 & 0.065303 & 0.392337 & 0.065305 \\
\hline 0.1 & 0.1 & 0.72 & 0.1 & 1.0 & 0.398723 & 0.063696 & 0.398724 & 0.063698 \\
\hline 0.1 & 0.1 & 0.72 & 0.1 & 2.0 & 0.408877 & 0.061176 & 0.408879 & 0.061177 \\
\hline
\end{tabular}

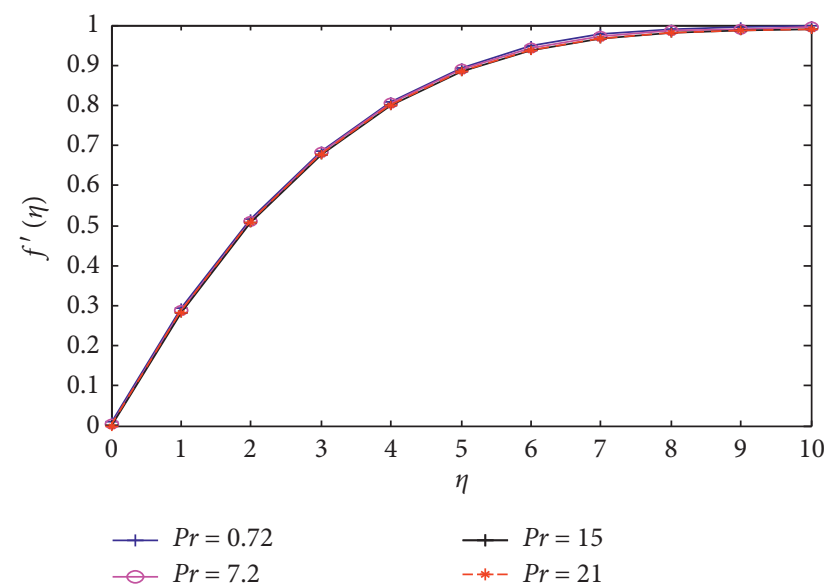

FIGURE 1: Velocity profiles for various values of the Prandtl number $(\operatorname{Pr})$ when $B i=0.1, G r=0.1, M=0.1, \lambda=0.1$, and $\mathrm{Ra}=0.1$

values for the Nusselt number $\left(-\theta^{\prime}(0)\right)$ and surface temperature $(\theta(0))$ for $\operatorname{Pr}=0.72$ and $G r=\lambda=R a=M=0$ is presented, and it is in an excellent agreement with the

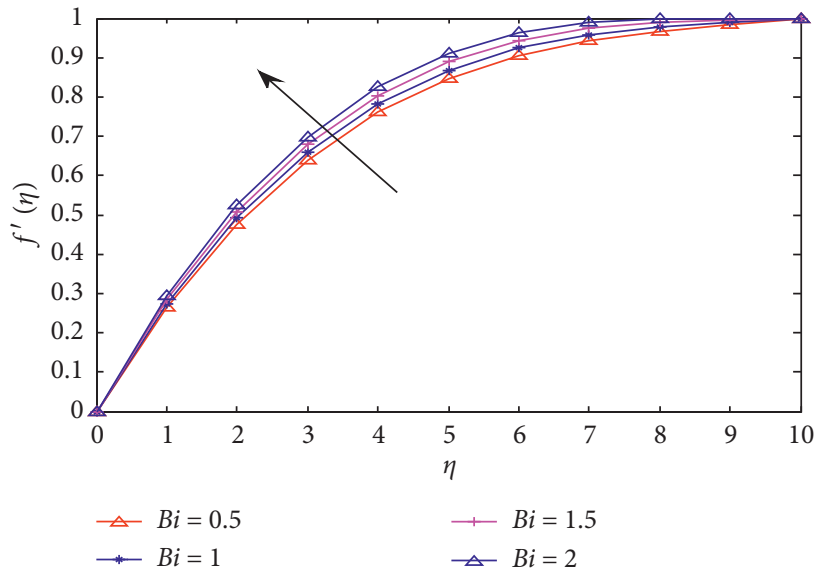

FIgURE 2: Velocity profiles for various values of the Biot number (Bi) when $\operatorname{Pr}=0.72, G r=0.1, M=0.1, \lambda=0.1$, and, $R a=0.1$.

corresponding values reported by Olanrewaju et al. [7], Aziz [22], Ishak [20], and Makinde and Oladapo Olanrewaju [24]. Table 2 shows a comparison of the values of the skin-friction 


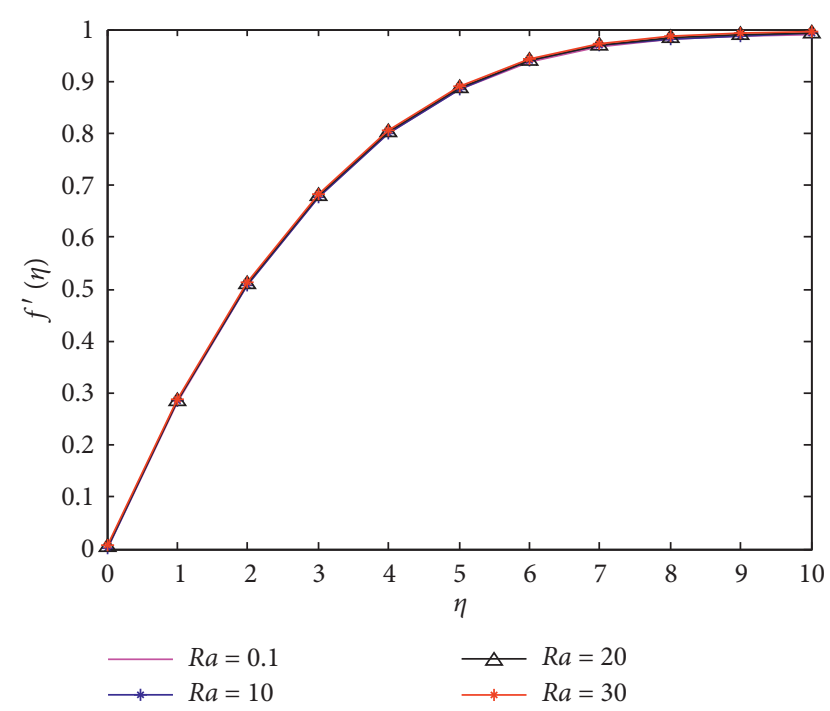

FIgURE 3: Velocity profiles for various values of the radiation parameter when $\operatorname{Pr}=0.72, G r=0.1, M=0.1, \lambda=0.1$, and $B i=0.1$.

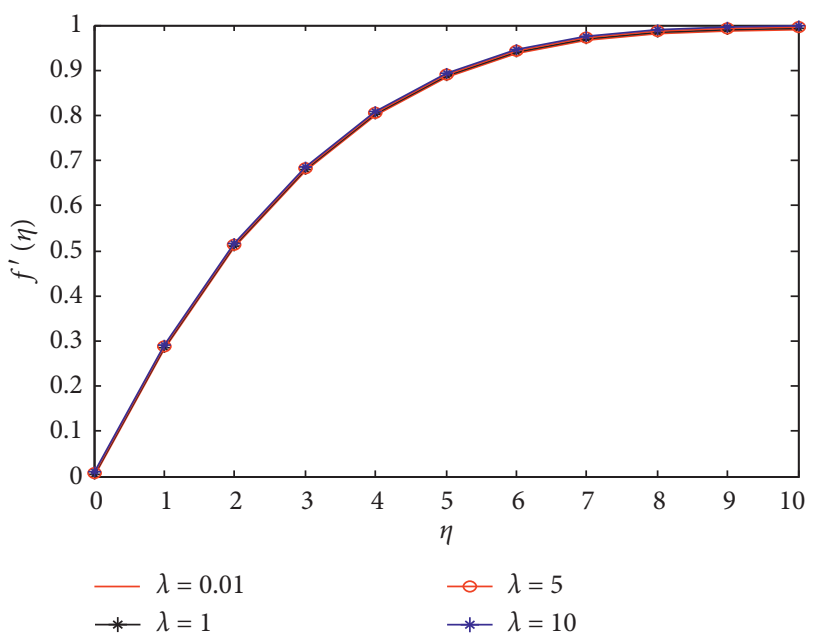

FIGURE 4: Velocity profiles for various values of the internal heat generation parameter $(\lambda)$ when $\operatorname{Pr}=0.72, \mathrm{Gr}=0.1, M=0.1, R a=0.1$, and $B i=0.1$.

coefficient $f^{\prime \prime}(0)$ and the local Nusselt number $\left(-\theta^{\prime}(0)\right)$ obtained by the OHAM in this study and the numerical method reported by Olanrewaju et al. [7] for various values of embedded parameters and shows an excellent agreement. As illustrated in Table 2, the skin friction and the rate of heat transfer at the plate surface increased with an increase in the local Grashof number, the internal heat generation parameter, the Biot number, and the radiation absorption parameter. However, an increase in the Prandtl number reduced the skin friction but increased the rate of heat transfer at the plate surface.

Figures 1-6 exhibit the effects of various parameters on the nondimensional velocity profiles within the boundary layer. Generally, the fluid velocity is zero at the plate surface and rises slowly away from the plate towards the free stream

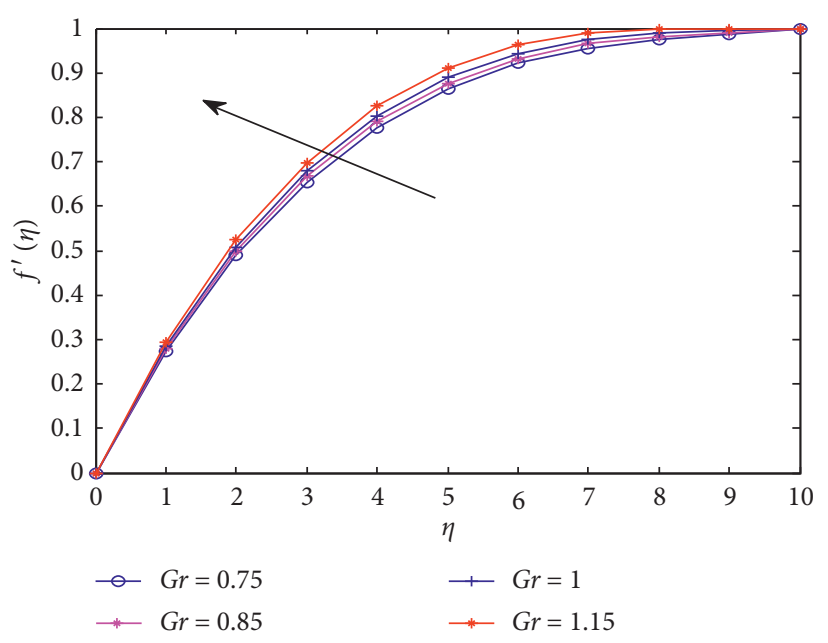

FIGURE 5: Velocity profiles for various values of the Grashof number $(G r)$ when $P r=0.72, \lambda=0.1, M=0.1, R a=0.1$, and $B i=0.1$.

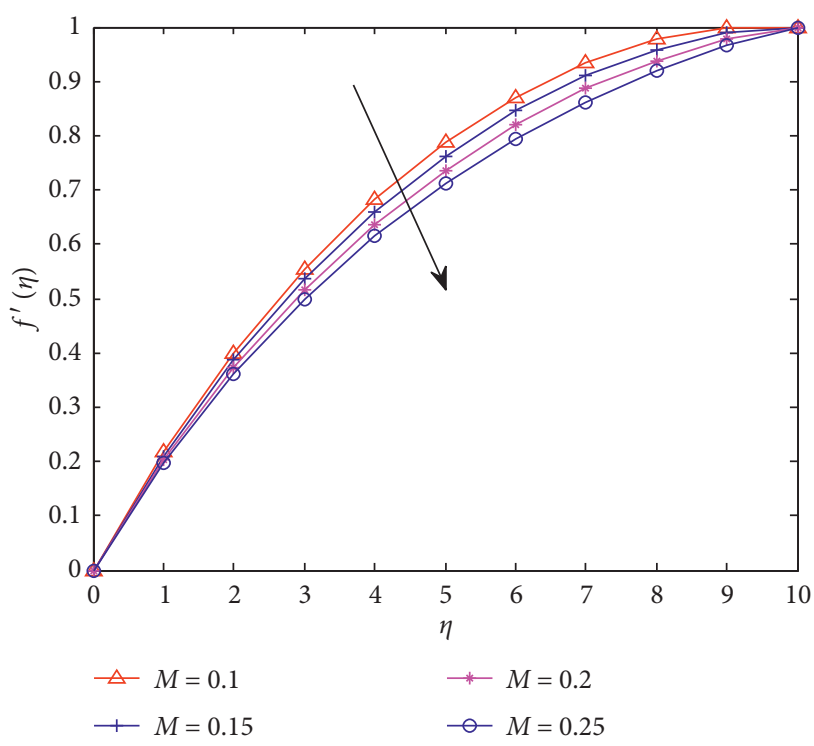

FIgURE 6: Velocity profiles for various values of the magnetic parameter $(M)$ when $P r=0.72, \lambda=0.1, \mathrm{Gr}=0.1, M=0.1, R a=0.1$, and $B i=0.1$.

value satisfying the boundary condition. The velocity profile for different values of the Prandtl number, thermal radiation, and internal heat generation is described in Figures 1, 3, and 4 , respectively. It is evident from the figures that the variation of values of the Prandtl number, internal heat generation, and radiation parameter had little effect or immaterial effect on the fluid velocity. Even though the velocity profile shows a bit decreasing trend as the value of the Prandtl number increases, it is insignificant. Similarly, from Figures 3 and 4, we see that the variation of values of the radiation parameter and internal heat generation has no significant effect even if the velocity profile shows a little increasing tendency in both cases. The effect of the Biot number and Grashof number on the fluid velocity is 


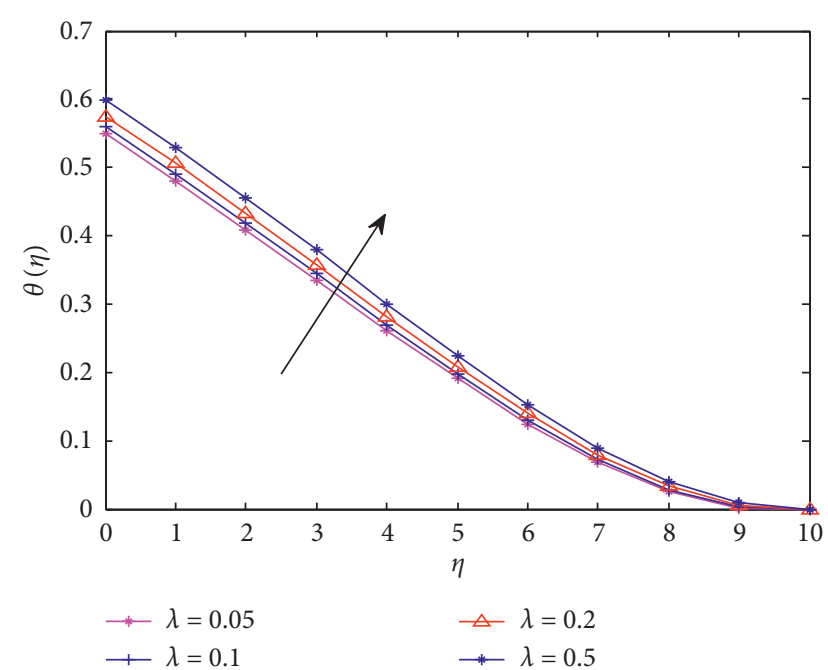

Figure 7: Temperature profiles for various values of internal heat generation $(\lambda)$ when $\operatorname{Pr}=0.72, M=0.1, G r=0.1, R a=0.1$, and $B i=0.1$.

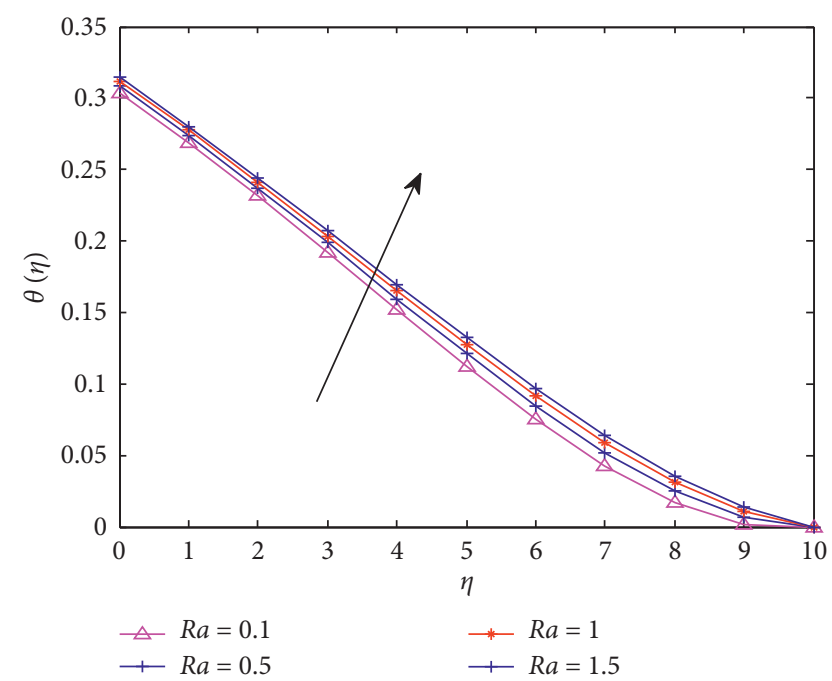

FIGURE 8: Temperature profiles for various values of the radiation parameter $(R a)$ when $\lambda=0.1, M=0.1, G r=0.1, P r=0.72$, and $B i=0.1$.

depicted in Figures 2 and 5, respectively. As one can observe from the figures, increase in the Biot number and Grashof number enhances the fluid velocity. In addition, the curves show that the peak value of velocity increases rapidly near the wall of the plate as the Grashof number increases, and it is understood that the rate of heat transfer at the plate surface increases with the increase of the Grashof number. Figure 6 reveals that an increase in the magnetic parameter results in the decrease of the fluid velocity. In reality, the existence of the magnetic field in an electrically conducting fluid introduces a force called Lorentz force which acts contrary to the flow if the magnetic field is applied normal to the fluid flow. This type of resistive force leads to slowing down of the flow field. Thus, our result and physical reality are consistent.

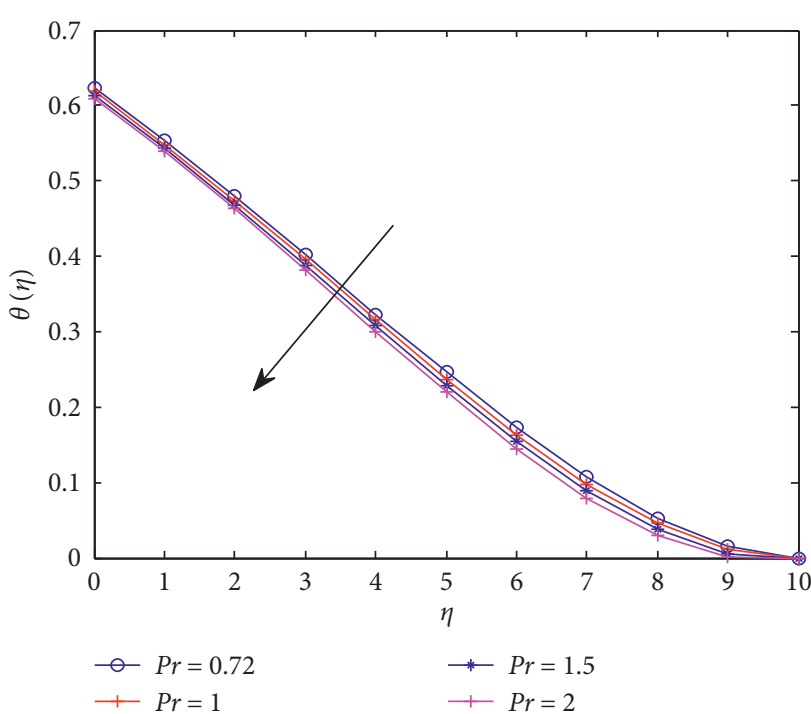

FIgure 9: Temperature profiles for various values of the Prandtl number $(P r)$ when $\lambda=0.1, M=0.1, G r=0.1, R a=0.1$, and $B i=0.1$.

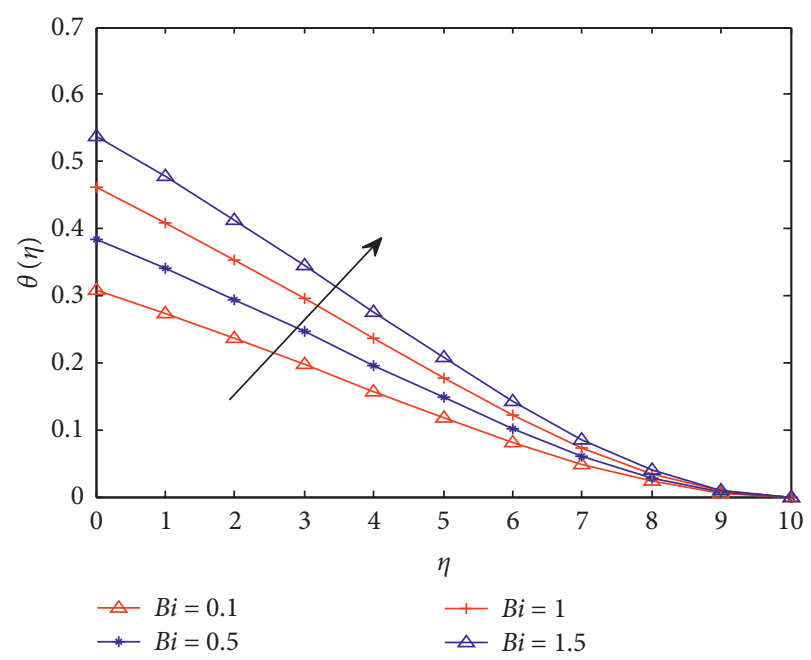

FIgURE 10: Temperature profiles for various values of the Biot number $(B i)$ when $\lambda=0.1, M=0.1, G r=0.1, R a=0.1$, and $P r=0.1$.

The influences of various embedded parameters on the fluid temperature within the boundary layer are illustrated in Figures 7-10. The figures illustrate the behaviour of the temperature profiles within the boundary layer. Commonly, at the plat surface, the fluid temperature is high and exponentially decreases to zero far away from the plate, satisfying boundary conditions. Figures 7 and 8 , respectively, demonstrate the behaviour of dimensionless temperature profiles for different values of internal heat generation $\lambda$ and the thermal radiation parameter, and it is seen that the temperature profile increases with the increase of some values of both internal heat generation and thermal radiation parameter. Therefore, the internal heat generation parameter and radiation parameter enhance 
the thermal diffusion within the boundary layer. It is seen from Figure 9 that the temperature profile decrease for increasing values of the Prandtl number at all points which indicates that thermal boundary layer thickness is reduced under the influence of the Prandtl number. Figure 10 illustrates that the Biot number causes the fluid temperature to increase. Generally, from these figures, it is notable that the thermal boundary layer thickness increased with an increase in the Biot number, internal heat generation, and thermal radiation but decreased with increasing values of the Grashof number, magnetic parameter, and Prandtl number. Thus, the convective surface heat transfer, the internal heat generation parameter, and the radiation parameter improved thermal diffusion, whereas an increase in the Prandtl number and the intensity of buoyancy force slowed down the rate of thermal diffusion within the boundary layer.

\section{Conclusion}

In this study, the optimal homotopy asymptotic method was successfully applied to investigate the effects of thermal radiation, internal heat generation, Prandtl number, Grashof number, and Biot number on the laminar boundary layer flow over a vertical plate in the presence of a convective surface boundary condition. The present analysis leads to the following conclusions:

(1) With the increase in the Grashof number, the fluid velocity and the values of the skin-friction coefficient increase, but the fluid temperature and the value of the Nusselt number reduce with the increase in the Grashof number.

(2) With the increase in the Prandtl number, the fluid temperature, the coefficient of skin friction, and heat transfer rate decrease, but the significant effect is not observed on the fluid velocity.

(3) The fluid velocity, temperature profile, and skinfriction coefficient increase with the increase in the Biot number.

(4) The fluid temperature, the values of the skin-friction coefficient, and the heat transfer rate increase with the increase of internal heat generation.

(5) With the increase of the radiation absorption parameter, the fluid temperature, the coefficient of skin friction, and the heat transfer rate enhance.

(6) Both fluid velocity and temperature profile fall with the increase in the magnetic parameter.

(7) The results confirm that the optimal homotopy asymptotic method is powerful in solving nonlinear differential problems.

\section{Data Availability}

The data used to support the findings of this study are available from the corresponding author upon request.

\section{Conflicts of Interest}

The authors declare that there are no conflicts of interest regarding the publication of this paper.

\section{Acknowledgments}

The authors express their heartfelt gratitude to the authors of literature studies used in this paper for the provided scientific aspects and idea that supplement this work.

\section{References}

[1] J. D. Anderson, "Ludwig prandtl's boundary layer," Physics Today, vol. 58, no. 12, pp. 42-48, 2005.

[2] H. Blasius, "Boundary layers in fluids of small viscosity," $Z$ Math Physik, vol. 56, no. 1, pp. 1-37, 1908.

[3] T. Fang, "Similarity solutions for a moving-flat plate thermal boundary layer," Acta Mechanica, vol. 163, no. 3-4, pp. 161-172, 2003.

[4] O. D. Makinde, "Free convection flow with thermal radiation and mass transfer past a moving vertical porous plate," International Communications in Heat and Mass Transfer, vol. 32, no. 10, pp. 1411-1419, 2005.

[5] R. Cortell, "Flow and heat transfer of a fluid through a porous medium over a stretching surface with internal heat generation/absorption and suction/blowing," Fluid Dynamics Research, vol. 37, no. 4, pp. 231-245, 2005.

[6] J.-J. Shu and I. Pop, "On thermal boundary layers on a flat plate subjected to a variable heat flux," International Journal of Heat and Fluid Flow, vol. 19, no. 1, pp. 79-84, 1998.

[7] P. O. Olanrewaju, J. A. Gbadeyan, T. Hayat, and A. A. Hendi, "Effects of internal heat generation, thermal radiation and buoyancy force on a boundary layer over a vertical plate with a convective surface boundary condition," South African Journal of Science, vol. 107, no. 9-10, pp. 80-85, 2011.

[8] D. J. Wanous and E. M. Sparrow, "Heat transfer for flow longitudinal to a cylinder with surface mass transfer," Journal of Heat Transfer, vol. 87, no. 2, pp. 317-319, 1965.

[9] Do Catherall, S. Keith, and P. G. Williams, "Viscous flow past a flat plate with uniform injection," Proceedings of the Royal Society of London. Series A. Mathematical and Physical Sciences, vol. 284, no. 1398, pp. 370-396, 1965.

[10] E. M. Sparrow, H. Quack, and C. J. Boerner, "Local nonsimilarity boundary-layer solutions," AIAA Journal, vol. 8, no. 11, pp. 1936-1942, 1970.

[11] M. Massoudi, "Local non-similarity solutions for the flow of a non-newtonian fluid over a wedge," International Journal of Non-linear Mechanics, vol. 36, no. 6, pp. 961-976, 2001.

[12] B. C. Sakiadis, "Boundary-layer behavior on continuous solid surfaces: I. Boundary-layer equations for two-dimensional and axisymmetric flow," AIChE Journal, vol. 7, no. 1, pp. 26-28, 1961.

[13] L. J. Crane, "Flow past a stretching plate," Zeitschrift für angewandte Mathematik und Physik ZAMP, vol. 21, no. 4, pp. 645-647, 1970.

[14] J. E. Danberg and K. S. Fansler, "A nonsimilar moving-wall boundary-layer problem," Quarterly of Applied Mathematics, vol. 34, no. 3, pp. 305-309, 1976.

[15] P. S. Gupta and A. S. Gupta, "Heat and mass transfer on a stretching sheet with suction or blowing," The Canadian 
Journal of Chemical Engineering, vol. 55, no. 6, pp. 744-746, 1977.

[16] K. Vijravelu, "Hydrodynamic flow and heat transfer over a continuous moving porous plate," Acta Mechanica, vol. 64, pp. 179-185, 1986.

[17] B. K. Dutta, "Heat transfer from a stretching sheet with uniform suction and blowing," Acta Mechanica, vol. 78, no. 34, pp. 255-262, 1989.

[18] A. Raptis, C. Perdikis, and H. S. Takhar, "Effect of thermal radiation on MHD flow," Applied Mathematics and Computation, vol. 153, no. 3, pp. 645-649, 2004.

[19] T. Hayat, Z. Abbas, M. Sajid, and S. Asghar, "The influence of thermal radiation on MHD flow of a second grade fluid," International Journal of Heat and Mass Transfer, vol. 50, no. 56, pp. 931-941, 2007.

[20] A. Ishak, "Similarity solutions for flow and heat transfer over a permeable surface with convective boundary condition," Applied Mathematics and Computation, vol. 217, no. 2, pp. 837-842, 2010.

[21] A. Aziz, "A similarity solution for laminar thermal boundary layer over a flat plate with a convective surface boundary condition," Communications in Nonlinear Science and $\mathrm{Nu}$ merical Simulation, vol. 14, no. 4, pp. 1064-1068, 2009.

[22] A. Aziz, "Hydrodynamic and thermal slip flow boundary layers over a flat plate with constant heat flux boundary condition," Communications in Nonlinear Science and Numerical Simulation, vol. 15, no. 3, pp. 573-580, 2010.

[23] P. Garg, G. N. Purohit, and R. C. Chaudhary, "A similarity solution for laminar thermal boundary layer over a flat plate with internal heat generation and a convective surface boundary condition," Journal of Rajasthan Academy of Physical Sciences, vol. 14, no. 2, pp. 221-226, 2015.

[24] O. D. Makinde and P. Oladapo Olanrewaju, "Buoyancy effects on thermal boundary layer over a vertical plate with a convective surface boundary condition," Journal of Fluids Engineering, vol. 132, no. 4, 2010.

[25] V. Marinca, N. Herişanu, C. Bota, and B. Marinca, "An optimal homotopy asymptotic method applied to the steady flow of a fourth-grade fluid past a porous plate," Applied Mathematics Letters, vol. 22, no. 2, pp. 245-251, 2009.

[26] V. Marinca and N. Herişanu, "Application of optimal homotopy asymptotic method for solving nonlinear equations arising in heat transfer," International Communications in Heat and Mass Transfer, vol. 35, no. 6, pp. 710-715, 2008.

[27] V. Marinca and N. Herisanu, Optimal Homotopy Asymptotic Method. in The Optimal Homotopy Asymptotic Method, pp. 9-22, Springer, Cham, Switzerland, 2015.

[28] F. Ghani, C. L. Ali, M. M. Rashidi, and T. Hajjari, "Application of modified optimal homotopy perturbation method to higher order boundary value problems in a finite domain," Hacettepe Journal of Mathematics and Statistics, vol. 45, no. 4, pp. 1049-1060, 2016.

[29] N. Herisanu, V. Marinca, G. Madescu, and F. Dragan, "Dynamic response of a permanent magnet synchronous generator to a wind gust," Energies, vol. 12, no. 5, p. 915, 2019.

[30] R. Nawaz, Z. Hussain, A. Khattak, and A. Khan, "Extension of optimal homotopy asymptotic method with use of daftardar-jeffery polynomials to coupled nonlinear korteweg-devries system," Complexity, vol. 2020, Article ID 6952709, 6 pages, 2020. 